\title{
Article \\ Multiple Diamond-Alpha Integral in General Form and Their Properties, Applications
}

\author{
Zhong-Xuan Mao, Ya-Ru Zhu *, Jun-Ping Hou, Chun-Ping Ma and Shi-Pu Liu
}

check for updates

Citation: Mao, Z.-X.; Zhu, Y.-R.; Hou, J.-P.; Ma, C.-P.; Liu, S.-P. Multiple Diamond-Alpha Integral in General Form and Their Properties, Applications. Mathematics 2021, 9, 1123. https://doi.org/10.3390/ math9101123

Academic Editor: Jaan Janno

Received: 3 April 2021

Accepted: 12 May 2021

Published: 15 May 2021

Publisher's Note: MDPI stays neutral with regard to jurisdictional claims in published maps and institutional affiliations.

Copyright: (C) 2021 by the authors. Licensee MDPI, Basel, Switzerland. This article is an open access article distributed under the terms and conditions of the Creative Commons Attribution (CC BY) license (https:// creativecommons.org/licenses/by/ $4.0 /)$.
Department of Mathematics and Physics, North China Electric Power University, Yonghua Street 619, Baoding 071003, China; maozhongxuan@ncepu.edu.cn (Z.-X.M.); jphou@ncepu.edu.cn (J.-P.H.); cpma@ncepu.edu.cn (C.-P.M.); spliu@ncepu.edu.cn (S.-P.L.)

* Correspondence: zhuyaru1982@ncepu.edu.cn; Tel.: +86-15131270870

\begin{abstract}
In this paper, we introduce the concept of $n$-dimensional Diamond-Alpha integral on time scales. In particular, it transforms into multiple Delta, Nabla and mixed integrals by taking different values of alpha. Some of its properties are explored, and the relationship between it and the multiple mixed integral is provided. As an application, we establish some weighted Ostrowski type inequalities through the new integral. These new inequalities expand some known inequalities in the monographs and papers, and in addition, furnish some other interesting inequalities. Examples of Ostrowski type inequalities are posed in detail at the end of the paper.
\end{abstract}

Keywords: multiple Diamond-Alpha integral; Ostrowski type inequalities; Delta integral; Nabla integral

MSC: Primary 26E70; 26E25

\section{Introduction}

Since Hilger [1] initiated the theory of time scales in 1988, it has been used widely by many branches of sciences. Moreover, many studies of the topic of time scales have been published, such as [2-15].

In [2], Bohner and Georgiev defined the following multiple Delta integral by using Riemann's definition of the integral,

$$
\int_{a_{n}}^{b_{n}} \int_{a_{n-1}}^{b_{n-1}} \cdots \int_{a_{1}}^{b_{1}} h\left(v_{1}, v_{2}, \cdots, v_{n}\right) \Delta_{1} v_{1} \Delta_{2} v_{2} \cdots \Delta_{n} v_{n} .
$$

Moreover, Bohner and Georgiev pointed out that multiple Nabla integral

$$
\int_{a_{n}}^{b_{n}} \int_{a_{n-1}}^{b_{n-1}} \cdots \int_{a_{1}}^{b_{1}} h\left(v_{1}, v_{2}, \cdots, v_{n}\right) \nabla_{1} v_{1} \nabla_{2} v_{2} \cdots \nabla_{n} v_{n},
$$

and mixed integrals that contain part of " $\Delta$ " and part of " $\nabla$ " can be defined in the same way.

As a linear combination of $\Delta$ and $\nabla$ dynamic derivatives, researchers introduced the Diamond-Alpha differentiation on time scales. More details about $\diamond_{\alpha}$ dynamic derivatives can be found in [16-19].

In [20], Tian, Zhu, and Cheung proposed the definition of multiple Diamond-Alpha integral

$$
\int_{a_{n}}^{b_{n}} \int_{a_{n-1}}^{b_{n-1}} \cdots \int_{a_{1}}^{b_{1}} h\left(v_{1}, v_{2}, \cdots, v_{n}\right) \diamond_{\alpha} v_{1} \diamond_{\alpha} v_{2} \cdots \diamond_{\alpha} v_{n},
$$

by employing antiderivatives of single-variable functions. 
In this paper we will consider the Diamond-Alpha integral of different alpha values, which is a more general form of (3), namely,

$$
\int_{a_{n}}^{b_{n}} \int_{a_{n-1}}^{b_{n-1}} \cdots \int_{a_{1}}^{b_{1}} h\left(v_{1}, v_{2}, \cdots, v_{n}\right) \diamond_{\alpha_{1}} v_{1} \diamond_{\alpha_{2}} v_{2} \cdots \diamond_{\alpha_{n}} v_{n}
$$

we call it multiple Diamond- $\alpha_{i}$ integral for convenience.

Ostrowski type inequality on time scales has received a lot attention [9,21-24]. In particular, the authors provided several Diamond-Alpha weighted Ostrowski type inequalities [21] as follows.

Theorem 1. If $\omega$ is a positive and continuous function, $\phi_{k}(k=1,2,3) \in C\left([a, b]_{\mathbb{T}}, \mathbb{R}\right)$ are bounded and satisfy

$$
\left\|\phi_{k}^{\Delta}\right\|_{\infty}=\sup _{x \in[a, b]}\left|\phi_{k}^{\Delta}(x)\right|<\infty, \quad\left\|\phi_{k}^{\nabla}\right\|_{\infty}=\sup _{x \in[a, b]}\left|\phi_{k}^{\nabla}(x)\right|<\infty, \quad k=1,2,3,
$$

then the following inequalities

$$
\begin{aligned}
& \left|\prod_{k=1}^{3} \phi_{k}(x)-\frac{1}{3 \int_{a}^{b} \omega(v) \diamond_{\alpha} v} \sum_{k=1}^{3}\left(\prod_{j \neq k} \phi_{j}(x) \int_{a}^{b} \omega(v) \phi_{k}(v) \diamond_{\alpha} v\right)\right| \\
\leq & \frac{1}{3 \int_{a}^{b} \omega(v) \diamond_{\alpha} v} \sum_{k=1}^{3}\left(\prod_{j \neq k}\left|\phi_{j}(x)\right|\left(\alpha\left\|\phi_{k}^{\Delta}\right\|_{\infty}+(1-\alpha)\left\|\phi_{k}^{\nabla}\right\|_{\infty}\right)\right) \int_{a}^{b} \omega(v)|x-v| \diamond_{\alpha} v,
\end{aligned}
$$

and

$$
\begin{aligned}
& \mid \int_{a}^{b} \omega(x) \prod_{k=1}^{3} \phi_{k}(x) \diamond_{\alpha} x \\
- & \frac{1}{3 \int_{a}^{b} \omega(v) \phi_{k}(v) \diamond_{\alpha} v} \sum_{k=1}^{3}\left(\int_{a}^{b} \omega(x) \prod_{j \neq k} \phi_{j}(x) \diamond_{\alpha} x \int_{a}^{b} \omega(x) \phi_{k}(x) \diamond_{\alpha} x\right) \mid \\
\leq & \frac{1}{3 \int_{a}^{b} \omega(v) \phi_{k}(v) \diamond_{\alpha} v} \times \\
& \int_{a}^{b} \omega(x) \sum_{k=1}^{3}\left(\prod_{j \neq k} \phi_{j}(x)\left(\alpha\left\|\phi_{k}^{\Delta}\right\|_{\infty}+(1-\alpha)\left\|\phi_{k}^{\nabla}\right\|_{\infty}\right)\right)\left(\int_{a}^{b} \omega(v)|x-v| \diamond_{\alpha} v\right) \diamond_{\alpha} x,
\end{aligned}
$$

hold for all $x \in[a, b]$.

Theorem 2. If $\omega$ is a positive and continuous function, $\phi_{k}(k=1,2) \in C\left([a, b]_{\mathbb{T}}, \mathbb{R}\right)$ are bounded and satisfy

$$
\left\|\phi_{k}^{\Delta}\right\|_{\infty}=\sup _{x \in[a, b]}\left|\phi_{k}^{\Delta}(x)\right|<\infty, \quad\left\|\phi_{k}^{\nabla}\right\|_{\infty}=\sup _{x \in[a, b]}\left|\phi_{k}^{\nabla}(x)\right|<\infty, \quad k=1,2,
$$

then the following inequalities

$$
\begin{aligned}
& \left|\int_{a}^{b} \omega(v)\left(\phi_{1}(x) \phi_{2}(x)-\phi_{1}(x) \phi_{2}(v)-\phi_{1}(v) \phi_{2}(x)+\phi_{1}(v) \phi_{2}(v)\right) \diamond_{\alpha} v\right| \\
\leq & \prod_{k=1}^{2}\left(\alpha\left\|\phi_{k}^{\Delta}\right\|_{\infty}+(1-\alpha)\left\|\phi_{k}^{\nabla}\right\|_{\infty}\right) \int_{a}^{b} \omega(v)|x-v|^{2} \diamond_{\alpha} v,
\end{aligned}
$$

and 


$$
\begin{aligned}
& \left|\int_{a}^{b} \omega(v) \diamond_{\alpha} v \int_{a}^{b} \omega(v) \phi_{1}(v) \phi_{2}(v) \diamond_{\alpha} v-\left(\int_{a}^{b} \omega(v) \phi_{1}(v) \diamond_{\alpha} v\right)\left(\int_{a}^{b} \omega(v) \phi_{2}(v) \diamond_{\alpha} v\right)\right| \\
& \leq \prod_{k=1}^{2}\left(\alpha\left\|\phi_{k}^{\Delta}\right\|_{\infty}+(1-\alpha)\left\|\phi_{k}^{\nabla}\right\|_{\infty}\right) \int_{a}^{b} \omega(x)\left(\int_{a}^{b} \omega(v)|x-v|^{2} \diamond_{\alpha} v\right) \diamond_{\alpha} x \\
& \text { hold for all } x \in[a, b] .
\end{aligned}
$$

The structure of the rest of this paper is as follows: we provide some preliminaries in Section 2 and consider the definition of multiple Diamond- $\alpha_{i}$ integral and its properties in Section 3. In Section 4, we generalize Theorems 1 and 2 to the inequalities involving $m$ functions; the inequalities involving $m$ functions and $n$ variables are given. Some examples and conclusions are presented in Sections 5 and 6, respectively.

\section{Preliminaries}

In this section, we list some common definitions and properties related to time scales; they can be found in [2].

Time scales are an arbitrary nonempty closed subset of the real number including set of real numbers $\mathbb{R}$, positive integer numbers $\mathbb{N}$, and denoted as $\mathbb{T}$. For more comprehensive knowledge about time scales, readers can consult $[2,4,6,8,11,18,25-28]$. In what follows, $\left[a_{i}, b_{i}\right]_{\mathbb{T}_{i}}$ bespeak $\left[a_{i}, b_{i}\right] \cap \mathbb{T}_{i}$ and we default $a_{i}, b_{i} \in \mathbb{T}_{i}$ for all $i=1,2, \cdots, n$ always.

Definition 1. For $h \in \mathbb{T}$, forward and backward jump operator $\sigma, \rho: \mathbb{T} \rightarrow \mathbb{T}$ are defined by

$$
\sigma(h)=\inf \{l \in \mathbb{T}: l>h\}, \text { and } \rho(h)=\sup \{l \in \mathbb{T}: l>h\},
$$

respectively.

Clearly, we have $\sigma(h) \geq h \geq \rho(h)$.

Definition 2. If $\phi: \mathbb{T} \rightarrow \mathbb{R}$ and for all given $\epsilon>0$ there exists a neighborhood $U$ and $\phi^{\Delta}(h), \phi^{\nabla}(h)$ of $h$ such that

$$
\left|\phi(\sigma(h))-\phi(l)-\phi^{\Delta}(h)(\sigma(h)-l)\right| \leq \epsilon|\sigma(h)-l|,
$$

and

$$
\left|\phi(\rho(h))-\phi(l)-\phi^{\nabla}(h)(\sigma(h)-l)\right| \leq \epsilon|\sigma(h)-l|,
$$

for all $l \in U$, then $\phi$ is respectively called Delta and Nabla differentiable on $\mathbb{T}^{k}$ and $\mathbb{T}_{k}$, where $\mathbb{T}_{k}$, $\mathbb{T}^{k}$ is defined as follows:

$$
\begin{gathered}
\mathbb{T}_{k}= \begin{cases}\mathbb{T} \backslash[\inf \mathbb{T}, \sigma(\inf \mathbb{T})), & \text { if } \inf \mathbb{T}>-\infty, \\
\mathbb{T}, & \text { if inf } \mathbb{T}=-\infty\end{cases} \\
\mathbb{T}_{k}= \begin{cases}\mathbb{T} \backslash(\rho(\sup \mathbb{T}), \sup \mathbb{T}), & \text { if } \sup \mathbb{T}<\infty, \\
\mathbb{T}, & \text { if } \sup \mathbb{T}=\infty .\end{cases}
\end{gathered}
$$

Definition 3. Let $\Phi, \phi: \mathbb{T} \rightarrow \mathbb{R}$. If $\Phi^{\Delta}(h)=\phi(h)$ holds for any $h \in \mathbb{T}^{k}$. Then $\Phi$ is called $a$ $\Delta$-antiderivative of $\phi$. Moreover

$$
\int_{\alpha}^{h} \phi(\tau) \Delta \tau=\Phi(h)-\Phi(\alpha) .
$$


If for any $h \in \mathbb{T}_{k}$ satisfies $\Phi^{\nabla}(h)=\phi(h)$. Then $\Phi$ is called $a \nabla$-antiderivative of $\phi$. Moreover

$$
\int_{\alpha}^{h} \phi(\tau) \nabla \tau=\Phi(h)-\Phi(\alpha) .
$$

Obviously, Delta, Nabla derivatives and integrals satisfy linearity.

Diamond-alpha derivative and integral are respectively defined as linear combination of $\Delta, \nabla$ dynamic derivatives and integrals.

Definition 4. If $\phi: \mathbb{T} \rightarrow \mathbb{R}$ is Delta and Nabla differentiable, then

$$
\phi^{\diamond}(h)=\alpha \phi^{\Delta}(h)+(1-\alpha) \phi^{\nabla}(h) .
$$

Definition 5. If $\phi: \mathbb{T} \rightarrow \mathbb{R}$ is continuous on $[\alpha, \beta]$, then

$$
\int_{\alpha}^{\beta} \phi(h) \diamond_{\alpha} h=\alpha \int_{\alpha}^{\beta} \phi(h) \Delta h+(1-\alpha) \int_{\alpha}^{\beta} \phi(h) \nabla h .
$$

Diamond-Alpha derivatives and integrals have the following qualities.

Proposition 1. If $\phi_{1}, \phi_{2}: \mathbb{T} \rightarrow \mathbb{R}$ are Delta and Nabla differentiable (so called Diamond-Alpha differentiable) and continuous functions, $k_{1}, k_{2} \in \mathbb{R}$, then

$$
\left(k_{1} \phi_{1}(h)+k_{2} \phi_{2}(h)\right)^{\diamond_{\alpha}}=k_{1} \phi_{1}^{\diamond_{\alpha}}(h)+k_{2} \phi_{2}^{\diamond_{\alpha}}(h),
$$

and

$$
\int_{\alpha}^{\beta}\left(k_{1} \phi_{1}(h)+k_{2} \phi_{2}(h)\right) \diamond_{\alpha} h=k_{1} \int_{\alpha}^{\beta} \phi_{1}(h) \diamond_{\alpha} h+k_{2} \int_{\alpha}^{\beta} \phi_{2}(h) \diamond_{\alpha} s .
$$

\section{Definition and Simple Properties}

For more comprehensive knowledge about time scales, readers can consult $[2,4,6,8,11,18,25-28]$.

Basically consistent with the definition process from [20], we suppose $D=\mathbb{T}_{1} \times$ $\mathbb{T}_{2} \times \cdots \times \mathbb{T}_{n}$ and $\inf \mathbb{T}_{i}=a_{i}$, sup $\mathbb{T}_{i}=b_{i}(i=1,2, \cdots, n)$. In what follows, $\left[a_{i}, b_{i}\right]_{\mathbb{T}_{i}}$ bespeak $\left[a_{i}, b_{i}\right] \cap \mathbb{T}_{i}$ and by default we always have $a_{i}, b_{i} \in \mathbb{T}_{i}$ for all $i=1,2, \cdots, n$. Every $\alpha_{i}(i=1,2, \cdots, n) \in[0,1]$.

We denote $\int_{a_{k}}^{b_{k}} Q_{k}\left(v_{1}, v_{2}, \cdots, v_{n}\right) \diamond_{\alpha_{k}} v_{k}$ as diamond- $\alpha_{k}$ integral with respect to $v_{k}$ $(k=1,2, \cdots, n)$. If there exist functions $Q_{1}, Q_{2}, \cdots, Q_{n}$ defined on $D$ such that

$$
\begin{aligned}
& Q_{k+1}\left(v_{1}, v_{2}, \cdots, v_{k-1}, b_{k+1}, v_{k+1}, \cdots, v_{n}\right)-Q_{k+1}\left(v_{1}, v_{2}, \cdots, v_{k-1}, a_{k+1}, v_{k+1}, \cdots, v_{n}\right) \\
= & \int_{a_{k+1}}^{b_{k+1}} Q_{k}\left(v_{1}, v_{2}, \cdots, v_{n}\right) \diamond_{\alpha_{k+1}} v_{k+1} \\
= & \alpha_{k+1} \int_{a_{k+1}}^{b_{k+1}} Q_{k}\left(v_{1}, v_{2}, \cdots, v_{n}\right) \Delta_{k+1} v_{k+1}+\left(1-\alpha_{k+1}\right) \int_{a_{k+1}}^{b_{k+1}} Q_{k}\left(v_{1}, v_{2}, \cdots, v_{n}\right) \nabla_{k+1} v_{k+1},
\end{aligned}
$$

for all $k=0,1,2, \cdots, n-1$, where we write $h\left(v_{1}, v_{2}, \cdots, v_{n}\right)$ as $Q_{0}\left(v_{1}, v_{2}, \cdots, v_{n}\right)$, then we define the multiple Diamond- $\alpha_{i}$ integral as follows 


$$
\begin{aligned}
& \int_{a_{n}}^{b_{n}} \int_{a_{n-1}}^{b_{n-1}} \cdots \int_{a_{1}}^{b_{1}} h\left(v_{1}, v_{2}, \cdots, v_{n}\right) \diamond_{\alpha_{1}} v_{1} \diamond_{\alpha_{2}} v_{2} \cdots \diamond_{\alpha_{n}} v_{n} \\
= & \int_{a_{n}}^{b_{n}} \int_{a_{n-1}}^{b_{n-1}} \cdots \int_{a_{2}}^{b_{2}} Q_{1}\left(b_{1}, v_{2}, \cdots, v_{n}\right)-Q_{1}\left(a_{1}, v_{2}, \cdots, v_{n}\right) \diamond_{\alpha_{2}} v_{2} \diamond_{\alpha_{3}} v_{3} \cdots \diamond_{\alpha_{n}} v_{n} \\
= & \int_{a_{n}}^{b_{n}} \int_{a_{n-1}}^{b_{n-1}} \cdots \int_{a_{3}}^{b_{3}} Q_{2}\left(b_{1}, b_{2}, \cdots, v_{n}\right)-Q_{2}\left(b_{1}, a_{2}, \cdots, v_{n}\right) \\
- & Q_{2}\left(a_{1}, b_{2}, \cdots, v_{n}\right)+Q_{2}\left(a_{1}, a_{2}, \cdots, v_{n}\right) \diamond_{\alpha_{3}} v_{3} \diamond_{\alpha_{4}}, \cdots 4 \diamond_{\alpha_{n}} v_{n} \\
= & \cdots \\
= & l_{n, 2^{n}-1} Q_{n}\left(b_{1}, b_{2}, \cdots, b_{n-1}, b_{n}\right)+l_{n, 2^{n}-2} Q_{n}\left(b_{1}, b_{2}, \cdots, b_{n-1}, a_{n}\right) \\
+ & l_{n, 2^{n}-3} Q_{n}\left(b_{1}, b_{2}, \cdots, a_{n-1}, b_{n}\right)+l_{n, 2^{n}-4} Q_{n}\left(b_{1}, b_{2}, \cdots, a_{n-1}, a_{n}\right) \\
+ & \cdots \\
+ & \left.l_{n, 3} Q_{n}\left(a_{1}, a_{2}, \cdots, b_{n-1}, b_{n}\right)+l_{n, 2} Q_{n}\left(a_{1}, a_{2}, \cdots, b_{n-1}, a_{n}\right)\right) \\
+ & l_{n, 1}\left(Q_{n}\left(a_{1}, a_{2}, \cdots, a_{n-1}, b_{n}\right)+l_{n, 0} Q_{n}\left(a_{1}, a_{2}, \cdots, a_{n-1}, a_{n}\right)\right)
\end{aligned}
$$

where $l_{i, j}$ is defined by recursive formula, $l_{0,0}=1$ and for all $m=0,1,2, \cdots, n$

$$
l_{m+1, v}= \begin{cases}l_{m, v}, & \text { if } v=0,1,2, \cdots, 2^{m}-1, \\ 1-l_{m, v}, & \text { if } v=2^{m}, 2^{m}+1, \cdots, 2^{m+1}-1 .\end{cases}
$$

If we default the following notations,

$$
\begin{aligned}
& I_{a}^{b}\left\{Q_{n}\right\}\left(2^{n}-1\right)=Q_{n}\left(b_{1}, b_{2}, \cdots, b_{n-1}, b_{n}\right), \quad I_{a}^{b}\left\{Q_{n}\right\}\left(2^{n}-2\right)=Q_{n}\left(b_{1}, b_{2}, \cdots, b_{n-1}, a_{n}\right), \\
& I_{a}^{b}\left\{Q_{n}\right\}\left(2^{n}-3\right)=Q_{n}\left(b_{1}, b_{2}, \cdots, a_{n-1}, b_{n}\right), \quad I_{a}^{b}\left\{Q_{n}\right\}\left(2^{n}-4\right)=Q_{n}\left(b_{1}, b_{2}, \cdots, a_{n-1}, a_{n}\right), \\
& \cdots \\
& I_{a}^{b}\left\{Q_{n}\right\}(3)=Q_{n}\left(a_{1}, a_{2}, \cdots, b_{n-1}, b_{n}\right), \quad I_{a}^{b}\left\{Q_{n}\right\}(2)=Q_{n}\left(a_{1}, a_{2}, \cdots, b_{n-1}, a_{n}\right), \\
& I_{a}^{b}\left\{Q_{n}\right\}(1)=Q_{n}\left(a_{1}, a_{2}, \cdots, a_{n-1}, b_{n}\right), \quad I_{a}^{b}\left\{Q_{n}\right\}(0)=Q_{n}\left(a_{1}, a_{2}, \cdots, a_{n-1}, a_{n}\right),
\end{aligned}
$$

then (5) transforms to

$$
\begin{aligned}
& \int_{a_{n}}^{b_{n}} \int_{a_{n-1}}^{b_{n-1}} \cdots \int_{a_{1}}^{b_{1}} h\left(v_{1}, v_{2}, \cdots, v_{n}\right) \diamond_{\alpha_{1}} v_{1} \diamond_{\alpha_{2}} v_{2} \cdots \diamond_{\alpha_{n}} v_{n} \\
= & \sum_{k=0}^{2^{n}-1} l_{n, k} I_{a}^{b}\left\{Q_{n}\right\}(k) .
\end{aligned}
$$

Next we give an example to show the new integral.

Example 1. If $h\left(v_{1}, v_{2}, \cdots, v_{n}\right)=v_{1} v_{2} \cdots v_{n}, \mathbb{T}_{i}=\mathbb{Z} \cap\left[a_{i}, b_{i}\right](i=1,2, \cdots, n)$ and $a_{i}, b_{i} \in$ $\mathbb{Z}(i=1,2, \cdots, n)$. Then

$$
\int_{a_{n}}^{b_{n}} \int_{a_{n-1}}^{b_{n-1}} \cdots \int_{a_{1}}^{b_{1}} v_{1} v_{2} \cdots v_{n} \diamond_{\alpha_{1}} v_{1} \diamond_{\alpha_{2}} v_{2} \cdots \diamond_{\alpha_{n}} v_{n}=\prod_{k=1}^{n} \frac{\left(b_{k}+a_{k}-2 \alpha_{k}+1\right)\left(b_{k}-a_{k}\right)}{2} .
$$

Proof. According to the definition of Diamond-Alpha integral, for all $c \in \mathbb{R}$, we have

$$
\begin{aligned}
\int_{a}^{b} c x \diamond_{\alpha} x & =c\left(\alpha \int_{a}^{b} x \Delta x+(1-\alpha) \int_{a}^{b} x \nabla x\right) \\
& =c\left(\alpha\left(\frac{b^{2}-b}{2}-\frac{a^{2}-a}{2}\right)+(1-\alpha)\left(\frac{b^{2}+b}{2}-\frac{a^{2}+a}{2}\right)\right) \\
& =\frac{c(b+a-2 \alpha+1)(b-a)}{2} .
\end{aligned}
$$


Using the above conclusion repeatedly, it leads to the desired equality.

$$
\begin{aligned}
& \int_{a_{n}}^{b_{n}} \int_{a_{n-1}}^{b_{n-1}} \cdots \int_{a_{1}}^{b_{1}} v_{1} v_{2} \cdots v_{n} \diamond_{\alpha_{1}} v_{1} \diamond_{\alpha_{2}} v_{2} \cdots \diamond_{\alpha_{n}} v_{n} \\
= & \int_{a_{n}}^{b_{n}} \int_{a_{n-1}}^{b_{n-1}} \cdots \int_{a_{2}}^{b_{2}} \frac{\left(b_{1}+a_{1}-2 \alpha_{1}+1\right)\left(b_{1}-a_{1}\right)}{2} v_{2} v_{3} \cdots v_{n} \diamond_{\alpha_{2}} v_{2} \diamond_{\alpha_{3}} v_{3} \cdots \diamond_{\alpha_{n}} v_{n} \\
= & \cdots \\
= & \prod_{k=1}^{n} \frac{\left(b_{k}+a_{k}-2 \alpha_{k}+1\right)\left(b_{k}-a_{k}\right)}{2}
\end{aligned}
$$

In particular, if we choose $\mathbb{T}_{i}=\mathbb{R} \cap\left[a_{i}, b_{i}\right](i=1,2, \cdots, n)$, then (4) changes into

$$
\int_{a_{n}}^{b_{n}} \int_{a_{n-1}}^{b_{n-1}} \cdots \int_{a_{1}}^{b_{1}} h\left(v_{1}, v_{2}, \cdots, v_{n}\right) \mathrm{d} v_{1} \mathrm{~d} v_{2} \cdots \mathrm{d} v_{n}
$$

If we let every $\alpha_{i}(i=1,2, \cdots, n)$ to be equal to 0 or 1 , then (4) changes into (1) or (2). If $\alpha_{1}=\alpha_{2}=\cdots=\alpha_{n}$, then (4) becomes (3). This shows (4) is a generalization of (1), (2) and (3).

Another powerful consequence of (4) is that it can represent multiple mixed integrals effectively. If every $\alpha_{i}=\omega_{i}=0$ or 1 , then the multiple mixed integral can be written as

$$
\int_{a_{n}}^{b_{n}} \int_{a_{n-1}}^{b_{n-1}} \cdots \int_{a_{1}}^{b_{1}} h\left(v_{1}, v_{2}, \cdots, v_{n}\right) \diamond_{\omega_{1}} v_{1} \diamond \omega_{2} v_{2} \cdots \diamond_{\omega_{n}} v_{n}
$$

For example, the following multiple mixed integral

$$
\int_{a_{4}}^{b_{4}} \int_{a_{3}}^{b_{3}} \int_{a_{2}}^{b_{2}} \int_{a_{1}}^{b_{1}} h\left(v_{1}, v_{2}, v_{3}, v_{4}\right) \Delta_{1} v_{1} \nabla_{2} v_{2} \nabla_{3} v_{3} \Delta_{4} v_{4}
$$

can be changed into

$$
\int_{a_{4}}^{b_{4}} \int_{a_{3}}^{b_{3}} \int_{a_{2}}^{b_{2}} \int_{a_{1}}^{b_{1}} h\left(v_{1}, v_{2}, v_{3}, v_{4}\right) \diamond_{1} v_{1} \diamond_{0} v_{2} \diamond_{0} v_{3} \diamond_{1} v_{4}
$$

For convenience, we define functions $\psi_{i}:\{0,1\} \rightarrow[0,1](i=1,2, \cdots, n)$ as follows,

$$
\psi_{i}(x)= \begin{cases}\alpha_{i}, & \text { if } x=0 \\ 1-\alpha_{i}, & \text { if } x=1 .\end{cases}
$$

The next theorem provides the link between multiple Diamond- $\alpha_{i}$ integral and multiple mixed integrals. It shows that the multiple Diamond- $\alpha_{i}$ integral is also a linear combination of multiple mixed integrals, similar to the Diamond- $\alpha$ integral of one variable.

Theorem 3. If $\phi\left(v_{1}, v_{2}, \cdots, v_{n}\right)$ is Diamond- $\alpha_{i}(i=1,2, \cdots, n)$ integrable on $D$, then

$$
\begin{aligned}
& \int_{a_{1}}^{b_{1}} \int_{a_{2}}^{b_{2}} \cdots \int_{a_{n}}^{b_{n}} \phi\left(v_{1}, v_{2}, \cdots, v_{n}\right) \diamond_{\alpha_{1}} v_{1} \diamond_{\alpha_{2}} v_{2} \cdots \diamond_{\alpha_{n}} v_{n} \\
= & \sum_{\omega_{1}, \cdots, \omega_{n}=0 \text { or } 1} \psi_{1}\left(\omega_{1}\right) \cdots \psi_{n}\left(\omega_{n}\right) \int_{a_{1}}^{b_{1}} \cdots \int_{a_{n}}^{b_{n}} \phi\left(v_{1}, \cdots, v_{n}\right) \diamond_{\omega_{1}} v_{1} \cdots \diamond_{\omega_{n}} v_{n},
\end{aligned}
$$

where $\omega_{1}, \cdots, \omega_{n}=0$ or 1 means all non-repetitive possibilities in binary and $\psi_{i}(i=1,2, \cdots, n)$ are defined by (9), that means there are $2^{n}$ total items in the right-hand side. 
Proof. According to the definition of Diamond $-\alpha_{i}(i=1,2, \cdots, n)$ integral, we have

$$
\begin{aligned}
& \int_{a_{n}}^{b_{n}} \int_{a_{n-1}}^{b_{n-1}} \cdots \int_{a_{1}}^{b_{1}} \phi\left(v_{1}, v_{2}, \cdots, v_{n}\right) \diamond_{\alpha_{1}} v_{1} \diamond_{\alpha_{2}} v_{2} \cdots \diamond_{\alpha_{n}} v_{n} \\
& =\int_{a_{n}}^{b_{n}} \int_{a_{n-1}}^{b_{n-1}} \cdots \int_{a_{2}}^{b_{2}}\left(\int_{a_{1}}^{b_{1}} \alpha_{1} \phi\left(v_{1}, v_{2}, \cdots, v_{n}\right) \Delta_{1} v_{1}\right. \\
& \left.+\int_{a_{1}}^{b_{1}}\left(1-\alpha_{1}\right) \phi\left(v_{1}, v_{2}, \cdots, v_{n}\right) \nabla_{1} v_{1}\right) \diamond_{\alpha_{2}} v_{2} \diamond_{\alpha_{3}} v_{3} \cdots \diamond_{\alpha_{n}} v_{n} \\
& =\int_{a_{n}}^{b_{n}} \int_{a_{n-1}}^{b_{n-1}} \cdots \int_{a_{3}}^{b_{3}}\left(\int_{a_{2}}^{b_{2}} \int_{a_{1}}^{b_{1}} \alpha_{1} \alpha_{2} \phi\left(v_{1}, v_{2}, \cdots, v_{n}\right) \Delta_{1} v_{1} \Delta_{2} v_{2}\right. \\
& +\int_{a_{2}}^{b_{2}} \int_{a_{1}}^{b_{1}} \alpha_{1}\left(1-\alpha_{2}\right) \phi\left(v_{1}, v_{2}, \cdots, v_{n}\right) \Delta_{1} v_{1} \nabla_{2} v_{2} \\
& +\int_{a_{2}}^{b_{2}} \int_{a_{1}}^{b_{1}}\left(1-\alpha_{1}\right) \alpha_{2} \phi\left(v_{1}, v_{2}, \cdots, v_{n}\right) \nabla_{1} v_{1} \Delta_{2} v_{2} \\
& \left.+\int_{a_{2}}^{b_{2}} \int_{a_{1}}^{b_{1}}\left(1-\alpha_{1}\right)\left(1-\alpha_{2}\right) \phi\left(v_{1}, v_{2}, \cdots, v_{n}\right) \nabla_{1} v_{1} \nabla_{2} v_{2}\right) \diamond_{\alpha_{3}} v_{3} \diamond_{\alpha_{4}} v_{4} \cdots \diamond_{\alpha_{n}} v_{n} \\
& =\cdots \\
& =\int_{a_{n}}^{b_{n}} \int_{a_{n-1}}^{b_{n-1}} \cdots \int_{a_{1}}^{b_{1}} \alpha_{1} \alpha_{2} \cdots \alpha_{n} \phi\left(v_{1}, v_{2}, \cdots, v_{n}\right) \Delta_{1} v_{1} \Delta_{2} v_{2} \cdots \Delta_{n} v_{n} \\
& +\int_{a_{n}}^{b_{n}} \int_{a_{n-1}}^{b_{n-1}} \cdots \int_{a_{1}}^{b_{1}} \alpha_{1} \alpha_{2} \cdots\left(1-\alpha_{n}\right) \phi\left(v_{1}, v_{2}, \cdots, v_{n}\right) \Delta_{1} v_{1} \Delta_{2} v_{2} \cdots \nabla_{n} v_{n} \\
& +\ldots \\
& +\int_{a_{n}}^{b_{n}} \int_{a_{n-1}}^{b_{n-1}} \cdots \int_{a_{1}}^{b_{1}}\left(1-\alpha_{1}\right)\left(1-\alpha_{2}\right) \cdots \alpha_{n} \phi\left(v_{1}, v_{2}, \cdots, v_{n}\right) \nabla_{1} v_{1} \nabla_{2} v_{2} \cdots \Delta_{n} v_{n} \\
& +\int_{a_{n}}^{b_{n}} \int_{a_{n-1}}^{b_{n-1}} \cdots \int_{a_{1}}^{b_{1}}\left(1-\alpha_{1}\right)\left(1-\alpha_{2}\right) \cdots\left(1-\alpha_{n}\right) \phi\left(v_{1}, v_{2}, \cdots, v_{n}\right) \nabla_{1} v_{1} \nabla_{2} v_{2} \cdots \nabla_{n} v_{n} \\
& =\sum_{\omega_{1}, \cdots, \omega_{n}=0 \text { or } 1} \psi_{1}\left(\omega_{1}\right) \cdots \psi_{n}\left(\omega_{n}\right) \int_{a_{n}}^{b_{n}} \cdots \int_{a_{1}}^{b_{1}} \phi\left(v_{1}, \cdots, v_{n}\right) \diamond_{\omega_{1}} v_{1} \cdots \diamond_{\omega_{n}} v_{n} .
\end{aligned}
$$

From the notations above, in [2], the following properties are introduced. These propositions show that multiple mixed integrals satisfy linearity and isotonicity.

Proposition 2 ([2], Theorem 7.50). Suppose $\phi_{1}, \phi_{2}$ is bounded, Delta and Nabla integrable on D, then the following formula holds

$$
\begin{aligned}
& \int_{a_{n}}^{b_{n}} \int_{a_{n-1}}^{b_{n-1}} \cdots \int_{a_{1}}^{b_{1}} \phi_{1}\left(v_{1}, v_{2}, \cdots, v_{n}\right)+\phi_{2}\left(v_{1}, v_{2}, \cdots, v_{n}\right) \diamond_{\omega_{1}} v_{1} \diamond_{\omega_{2}} v_{2} \cdots \diamond_{\omega_{n}} v_{n} \\
= & \int_{a_{n}}^{b_{n}} \int_{a_{n-1}}^{b_{n-1}} \cdots \int_{a_{1}}^{b_{1}} \phi_{1}\left(v_{1}, v_{2}, \cdots, v_{n}\right) \diamond_{\omega_{1}} v_{1} \diamond_{\omega_{2}} v_{2} \cdots \diamond_{\omega_{n}} v_{n} \\
+ & \int_{a_{n}}^{b_{n}} \int_{a_{n-1}}^{b_{n-1}} \cdots \int_{a_{1}}^{b_{1}} \phi_{2}\left(v_{1}, v_{2}, \cdots, v_{n}\right) \diamond_{\omega_{1}} v_{1} \diamond_{\omega_{2}} v_{2} \cdots \diamond_{\omega_{n}} v_{n}
\end{aligned}
$$

where $\omega_{1}, \cdots, \omega_{n}=0$ or 1 . 
Proposition 3 (See [2], Theorem 7.49). Suppose that the bounded function $\phi$ is Delta and Nabla integrable on $D$, then for all $k \in \mathbb{R}$

$$
\begin{aligned}
& \int_{a_{n}}^{b_{n}} \int_{a_{n-1}}^{b_{n-1}} \cdots \int_{a_{1}}^{b_{1}} k \phi\left(v_{1}, v_{2}, \cdots, v_{n}\right) \diamond_{\omega_{1}} v_{1} \diamond_{\omega_{2}} v_{2} \cdots \diamond_{\omega_{n}} v_{n} \\
= & k \int_{a_{n}}^{b_{n}} \int_{a_{n-1}}^{b_{n-1}} \cdots \int_{a_{1}}^{b_{1}} \phi\left(v_{1}, v_{2}, \cdots, v_{n}\right) \diamond_{\omega_{1}} v_{1} \diamond_{\omega_{2}} v_{2} \cdots \diamond_{\omega_{n}} v_{n},
\end{aligned}
$$

where $\omega_{1}, \cdots, \omega_{n}=0$ or 1 .

Corollary 1 ([2], Corollary 7.51). If $\phi_{1}, \phi_{2}, \cdots, \phi_{m}$ are bounded Delta and Nabla integrable over $D$, and $k_{1}, k_{2}, \cdots, k_{m} \in \mathbb{R}$, then

$$
\begin{aligned}
& \int_{a_{n}}^{b_{n}} \int_{a_{n-1}}^{b_{n-1}} \cdots \int_{a_{1}}^{b_{1}} \sum_{j=1}^{m} k_{j} \phi_{j}\left(v_{1}, v_{2}, \cdots, v_{n}\right) \diamond_{\omega_{1}} v_{1} \diamond_{\omega_{2}} v_{2} \cdots \diamond_{\omega_{n}} v_{n} \\
= & \sum_{j=1}^{m} \int_{a_{n}}^{b_{n}} \int_{a_{n-1}}^{b_{n-1}} \cdots \int_{a_{1}}^{b_{1}} k_{j} \phi_{j}\left(v_{1}, v_{2}, \cdots, v_{n}\right) \diamond_{\omega_{1}} v_{1} \diamond_{\omega_{2}} v_{2} \cdots \diamond_{\omega_{n}} v_{n},
\end{aligned}
$$

where $\omega_{1}, \cdots, \omega_{n}=0$ or 1 .

Proposition 4 ([2], Theorem 7.52). Suppose $\phi_{1}, \phi_{2}$ is bounded Delta and Nabla integrable on $D$, and $\phi_{1}\left(v_{1}, v_{2}, \cdots, v_{n}\right) \geq \phi_{2}\left(v_{1}, v_{2}, \cdots, v_{n}\right)$ holds on for all points in $D$, then

$$
\begin{aligned}
& \int_{a_{n}}^{b_{n}} \int_{a_{n-1}}^{b_{n-1}} \cdots \int_{a_{1}}^{b_{1}} \phi_{1}\left(v_{1}, v_{2}, \cdots, v_{n}\right) \diamond_{\omega_{1}} v_{1} \diamond_{\omega_{2}} v_{2} \cdots \diamond_{\omega_{n}} v_{n} \\
\geq & \int_{a_{n}}^{b_{n}} \int_{a_{n-1}}^{b_{n-1}} \cdots \int_{a_{1}}^{b_{1}} \phi_{2}\left(v_{1}, v_{2}, \cdots, v_{n}\right) \diamond_{\omega_{1}} v_{1} \diamond_{\omega_{2}} v_{2} \cdots \diamond_{\omega_{n}} v_{n},
\end{aligned}
$$

where $\omega_{1}, \cdots, \omega_{n}=0$ or 1 .

Remark 1. In fact, [2] just narrated the properties of multiple Delta integrals. However, since in the end of Chapter 7, the authors mentioned that multiple Nabla integrals and multiple mixed integrals should have the same properties, we give the general forms of the theorems in [2].

Next, we explore properties of (4) based on the Properties 2-4 and Theorem 3. We find that our new integrals also satisfy linearity and isotonicity.

Proposition 5. Suppose $\phi_{1}, \phi_{2}$ is bounded Diamond $-\alpha_{i}(i=1,2, \cdots, n)$ integrable on $D$, then we have

$$
\begin{aligned}
& \int_{a_{n}}^{b_{n}} \int_{a_{n-1}}^{b_{n-1}} \cdots \int_{a_{1}}^{b_{1}}\left(\phi_{1}\left(v_{1}, v_{2}, \cdots, v_{n}\right)+\phi_{2}\left(v_{1}, v_{2}, \cdots, v_{n}\right)\right) \diamond_{\alpha_{1}} v_{1} \diamond_{\alpha_{2}} v_{2} \cdots \diamond_{\alpha_{n}} v_{n} \\
= & \int_{a_{n}}^{b_{n}} \int_{a_{n-1}}^{b_{n-1}} \cdots \int_{a_{1}}^{b_{1}} \phi_{1}\left(v_{1}, v_{2}, \cdots, v_{n}\right) \diamond_{\alpha_{1}} v_{1} \diamond_{\alpha_{2}} v_{2} \cdots \diamond_{\alpha_{n}} v_{n} \\
+ & \int_{a_{n}}^{b_{n}} \int_{a_{n-1}}^{b_{n-1}} \cdots \int_{a_{1}}^{b_{1}} \phi_{2}\left(v_{1}, v_{2}, \cdots, v_{n}\right) \diamond_{\alpha_{1}} v_{1} \diamond_{\alpha_{2}} v_{2} \cdots \diamond_{\alpha_{n}} v_{n} .
\end{aligned}
$$


Proof. According to Proposition 2 and Theorem 3, we obtain

$$
\begin{aligned}
& \int_{a_{n}}^{b_{n}} \int_{a_{n-1}}^{b_{n-1}} \cdots \int_{a_{1}}^{b_{1}} \phi_{1}\left(v_{1}, v_{2}, \cdots, v_{n}\right)+\phi_{2}\left(v_{1}, v_{2}, \cdots, v_{n}\right) \diamond_{\alpha_{1}} v_{1} \diamond_{\alpha_{2}} v_{2} \cdots \diamond_{\alpha_{n}} v_{n} \\
= & \sum_{\omega_{1}, \cdots, \omega_{n}=0 \text { or } 1} \psi_{1}\left(\omega_{1}\right) \psi_{2}\left(\omega_{2}\right) \cdots \psi_{n}\left(\omega_{n}\right) \\
= & \int_{a_{n}}^{b_{n}} \int_{a_{n-1}}^{b_{n-1}} \cdots \int_{a_{1}}^{b_{1}} \phi_{1}\left(v_{1}, \cdots, v_{n}\right)+\phi_{2}\left(v_{1}, \cdots, v_{n}\right) \diamond_{\omega_{1}} v_{1} \diamond_{\omega_{2}} v_{2} \cdots \diamond_{\omega_{n}} v_{n} \\
& \sum_{\omega_{1}, \cdots, \omega_{n}=0 \text { or } 1} \psi_{1}\left(\omega_{1}\right) \psi_{2}\left(\omega_{2}\right) \cdots \psi_{n}\left(\omega_{n}\right) \\
& \left(\int_{a_{n}}^{b_{n}} \int_{a_{n-1}}^{b_{n-1}} \cdots \int_{a_{1}}^{b_{1}} \phi_{1}\left(v_{1}, v_{2}, \cdots, v_{n}\right) \diamond_{\omega_{1}} v_{1} \diamond_{\omega_{2}} v_{2} \cdots \diamond_{\omega_{n}} v_{n}\right. \\
& \left.+\int_{a_{n}}^{b_{n}} \int_{a_{n-1}}^{b_{n-1}} \cdots \int_{a_{1}}^{b_{1}} \phi_{2}\left(v_{1}, v_{2}, \cdots, v_{n}\right) \diamond_{\omega_{1}} v_{1} \diamond_{\omega_{2}} v_{2} \cdots \diamond_{\omega_{n}} v_{n}\right) \\
= & \left(\sum_{\omega_{1}, \cdots, \omega_{n}=0 \text { or } 1} \psi_{1}\left(\omega_{1}\right) \psi_{2}\left(\omega_{2}\right) \cdots \psi_{n}\left(\omega_{n}\right)\right. \\
& \left.\int_{a_{n}}^{b_{n}} \int_{a_{n-1}}^{b_{n-1}} \cdots \int_{a_{1}}^{b_{1}} \phi_{1}\left(v_{1}, v_{2}, \cdots, v_{n}\right) \diamond_{\omega_{1}} v_{1} \diamond_{\omega_{2}} v_{2} \cdots \diamond_{\omega_{n}} v_{n}\right) \\
+ & \left(\sum_{\omega_{1}, \cdots, \omega_{n}=0 \text { or } 1} \psi_{1}\left(\omega_{1}\right) \psi_{2}\left(\omega_{2}\right) \cdots \psi_{n}\left(\omega_{n}\right)\right. \\
& \left.\int_{a_{n}}^{b_{n}} \int_{a_{n-1}}^{b_{n-1}} \cdots \int_{a_{1}}^{b_{1}} \phi_{2}\left(v_{1}, v_{2}, \cdots, v_{n}\right) \diamond_{\omega_{1}} v_{1} \diamond_{\omega_{2}} v_{2} \cdots \diamond_{\omega_{n}} v_{n}\right) \\
= & \int_{a_{n}}^{b_{n}} \int_{a_{n-1}}^{b_{n-1}} \cdots \int_{a_{1}}^{b_{1}} \phi_{1}\left(v_{1}, v_{2}, \cdots, v_{n}\right) \diamond_{\alpha_{1}} v_{1} \diamond_{\alpha_{2}} v_{2} \cdots \diamond_{\alpha_{n}} v_{n} \\
+ & \int_{a_{n}}^{b_{n}} \int_{a_{n-1}}^{b_{n-1}} \cdots \int_{a_{1}}^{b_{1}} \phi_{2}\left(v_{1}, v_{2}, \cdots, v_{n}\right) \diamond_{\alpha_{1}} v_{1} \diamond_{\alpha_{2}} v_{2} \cdots \diamond_{\alpha_{n}} v_{n} .
\end{aligned}
$$

Therefore, the proof is complete.

Proposition 6. If $\phi\left(v_{1}, v_{2}, \cdots, v_{n}\right)$ is bounded Diamond- $\alpha_{i}(i=1,2, \cdots, n)$ integrable on $D$ and $k \in \mathbb{R}$, then

$$
\begin{aligned}
& \int_{a_{n}}^{b_{n}} \int_{a_{n-1}}^{b_{n-1}} \cdots \int_{a_{1}}^{b_{1}} k \phi\left(v_{1}, v_{2}, \cdots, v_{n}\right) \diamond_{\alpha_{1}} v_{1} \diamond_{\alpha_{2}} v_{2} \cdots \diamond_{\alpha_{n}} v_{n} \\
= & k \int_{a_{n}}^{b_{n}} \int_{a_{n-1}}^{b_{n-1}} \cdots \int_{a_{1}}^{b_{1}} \phi\left(v_{1}, v_{2}, \cdots, v_{n}\right) \diamond_{\alpha_{1}} v_{1} \diamond_{\alpha_{2}} v_{2} \cdots \diamond_{\alpha_{n}} v_{n} .
\end{aligned}
$$

The proof of Proposition 6 is similar to the proof of Proposition 5, we will not repeat it. Combining (10) and (11), we have the following corollary.

Corollary 2. If $\phi_{1}, \phi_{2}, \cdots, \phi_{m}$ is bounded Diamond- $\alpha_{i}(i=1,2, \cdots, n)$ integrable on $D$ and $k_{i} \in \mathbb{R}(i=1,2, \cdots, m)$, then

$$
\begin{aligned}
& \int_{a_{n}}^{b_{n}} \int_{a_{n-1}}^{b_{n-1}} \cdots \int_{a_{1}}^{b_{1}} \sum_{j=1}^{m} k_{j} \phi_{j}\left(v_{1}, v_{2}, \cdots, v_{n}\right) \diamond_{\alpha_{1}} v_{1} \diamond_{\alpha_{2}} v_{2} \cdots \diamond_{\alpha_{n}} v_{n} \\
= & \sum_{j=1}^{m} k_{j} \int_{a_{n}}^{b_{n}} \int_{a_{n-1}}^{b_{n-1}} \cdots \int_{a_{1}}^{b_{1}} \phi_{j}\left(v_{1}, v_{2}, \cdots, v_{n}\right) \diamond_{\alpha_{1}} v_{1} \diamond_{\alpha_{2}} v_{2} \cdots \diamond_{\alpha_{n}} v_{n} .
\end{aligned}
$$


Proposition 7. If $\phi_{1}, \phi_{2}$ is bounded Diamond- $\alpha_{i}(i=1,2, \cdots, n)$ integrable on $D$, and $\phi_{1}\left(v_{1}, v_{2}, \cdots, v_{n}\right) \geq \phi_{2}\left(v_{1}, v_{2}, \cdots, v_{n}\right)$ holds for all points in $D$, then

$$
\begin{aligned}
& \int_{a_{n}}^{b_{n}} \int_{a_{n-1}}^{b_{n-1}} \cdots \int_{a_{1}}^{b_{1}} \phi_{1}\left(v_{1}, v_{2}, \cdots, v_{n}\right) \diamond_{\alpha_{1}} v_{1} \diamond_{\alpha_{2}} v_{2} \cdots \diamond_{\alpha_{n}} v_{n} \\
\geq & \int_{a_{n}}^{b_{n}} \int_{a_{n-1}}^{b_{n-1}} \cdots \int_{a_{1}}^{b_{1}} \phi_{2}\left(v_{1}, v_{2}, \cdots, v_{n}\right) \diamond_{\alpha_{1}} v_{1} \diamond_{\alpha_{2}} v_{2} \cdots \diamond_{\alpha_{n}} v_{n} .
\end{aligned}
$$

In particular, if $\phi_{2}\left(v_{1}, v_{2}, \cdots, v_{n}\right) \equiv 0$, then we have the following corollary.

Corollary 3. If $\phi$ is bounded Diamond- $\alpha_{i}(i=1,2, \cdots, n)$ integrable on $D$, and $\phi\left(v_{1}, v_{2}, \cdots, v_{n}\right) \geq$ 0 holds for all points in $D$, then

$$
\int_{a_{n}}^{b_{n}} \int_{a_{n-1}}^{b_{n-1}} \cdots \int_{a_{1}}^{b_{1}} \phi\left(v_{1}, v_{2}, \cdots, v_{n}\right) \diamond_{\alpha_{1}} v_{1} \diamond_{\alpha_{2}} v_{2} \cdots \diamond_{\alpha_{n}} v_{n} \geq 0
$$

\section{Ostroski Type Inequalities on Multiple Diamond- $\alpha_{i}$ Integrals}

In this section, we establish some Ostrowski type inequalities through multiple Diamond- $\alpha_{i}$ integral. Specifically, we consider Ostrowski type inequalities with more functions and more variables. Theorem 1 just considered three functions, in fact, we found it is also holds for the case with $m$ functions. Thus, we have the following theorem.

Theorem 4. If $\lambda$ is a positive and continuous function, $\phi_{k}(k=1,2, \cdots, m) \in C\left([a, b]_{\mathbb{T}}, \mathbb{R}\right)$ are bounded, nonzero and satisfy

$$
\left\|\phi_{k}^{\Delta}\right\|_{\infty}:=\sup _{x \in[a, b]}\left|\phi_{k}^{\Delta}(x)\right|<\infty, \quad\left\|\phi_{k}^{\nabla}\right\|_{\infty}:=\sup _{x \in[a, b]}\left|\phi_{k}^{\nabla}(x)\right|<\infty, \quad k=1,2, \cdots, m,
$$

for given $m \geq 2$, then the following inequalities

$$
\begin{aligned}
& \left|\prod_{k=1}^{m} \phi_{k}(x)-\frac{1}{m \int_{a}^{b} \lambda(y) \diamond_{\alpha} y} \sum_{k=1}^{m}\left(\prod_{j \neq k} \phi_{j}(x) \int_{a}^{b} \lambda(y) \phi_{k}(y) \diamond_{\alpha} y\right)\right| \\
\leq & \frac{1}{m \int_{a}^{b} \lambda(y) \diamond_{\alpha} y} \sum_{k=1}^{m}\left(\prod_{j \neq k}\left|\phi_{j}(x)\right|\left(\alpha\left\|\phi_{k}^{\Delta}\right\|_{\infty}+(1-\alpha)\left\|\phi_{k}^{\nabla}\right\|_{\infty}\right)\right) \int_{a}^{b} \lambda(y)|x-y| \diamond_{\alpha} y,
\end{aligned}
$$

and

$$
\begin{aligned}
& \mid m \int_{a}^{b} \lambda(y) \phi_{k}(y) \diamond_{\alpha} y \int_{a}^{b} \lambda(x) \prod_{k=1}^{m} \phi_{k}(x) \diamond_{\alpha} x \\
- & \sum_{k=1}^{m}\left(\int_{a}^{b} \lambda(x) \prod_{j \neq k} \phi_{j}(x) \diamond_{\alpha} x \int_{a}^{b} \lambda(x) \phi_{k}(x) \diamond_{\alpha} x\right) \mid \\
\leq & \int_{a}^{b} \lambda(x) \sum_{k=1}^{m}\left(\prod_{j \neq k} \phi_{j}(x)\left(\alpha\left\|\phi_{k}^{\Delta}\right\|_{\infty}+(1-\alpha)\left\|\phi_{k}^{\nabla}\right\|_{\infty}\right)\right)\left(\int_{a}^{b} \lambda(y)|x-y| \diamond_{\alpha} y\right) \diamond_{\alpha} x,
\end{aligned}
$$

hold for all $x \in[a, b]$.

Proof. It is easy to check

$$
\begin{aligned}
\phi_{k}(x)-\phi_{k}(y) & =\int_{x}^{y} \phi_{k}^{\Delta}(v) \Delta v=\int_{x}^{y} \phi_{k}^{\nabla}(v) \nabla v \\
& =\alpha \int_{x}^{y} \phi_{k}^{\Delta}(v) \Delta v+(1-\alpha) \int_{x}^{y} \phi_{k}^{\nabla}(v) \nabla v, \quad k=1,2, \cdots, m .
\end{aligned}
$$


Then we have

$$
\begin{aligned}
& \phi_{k}(x) \prod_{j \neq k} \phi_{j}(x)-\phi_{k}(y) \prod_{j \neq k} \phi_{j}(x)=\prod_{k=1}^{m} \phi_{k}(x)-\phi_{k}(y) \prod_{j \neq k} \phi_{j}(x) \\
= & \prod_{j \neq k} \phi_{j}(x)\left(\alpha \int_{x}^{y} \phi_{k}^{\Delta}(v) \Delta v+(1-\alpha) \int_{x}^{y} \phi_{k}^{\nabla}(v) \nabla v\right), \quad k=1,2, \cdots, m .
\end{aligned}
$$

By adding up from $k=1$ to $m$, we can obtain

$$
\begin{aligned}
& m \prod_{k=1}^{m} \phi_{k}(x)-\sum_{k=1}^{m} \prod_{j \neq k} \phi_{j}(x) \phi_{k}(y) \\
= & \sum_{k=1}^{m} \prod_{j \neq k} \phi_{j}(x)\left(\alpha \int_{x}^{y} \phi_{k}^{\Delta}(v) \Delta v+(1-\alpha) \int_{x}^{y} \phi_{k}^{\nabla}(v) \nabla v\right),
\end{aligned}
$$

namely,

$$
\begin{aligned}
& m \prod_{k=1}^{m} \phi_{k}(x) \lambda(y)-\sum_{k=1}^{m} \prod_{j \neq k} \phi_{j}(x) \phi_{k}(y) \lambda(y) \\
= & \sum_{k=1}^{m} \prod_{j \neq k} \phi_{j}(x)\left(\alpha \int_{x}^{y} \phi_{k}^{\Delta}(v) \Delta v+(1-\alpha) \int_{x}^{y} \phi_{k}^{\nabla}(v) \nabla v\right) \lambda(y) .
\end{aligned}
$$

Diamond-Alpha integrating on both side from $a$ to $b$ with respect to $y$ and rewriting, we get

$$
\begin{aligned}
& \prod_{k=1}^{m} \phi_{k}(x)-\frac{1}{m \int_{a}^{b} \lambda(y) \diamond_{\alpha} y} \sum_{k=1}^{m} \prod_{j \neq k} \phi_{j}(x) \int_{a}^{b} \phi_{k}(y) \lambda(y) \diamond_{\alpha} y \\
= & \frac{1}{m \int_{a}^{b} \lambda(y) \diamond_{\alpha} y} \sum_{k=1}^{m} \prod_{j \neq k} \phi_{j}(x) \int_{a}^{b}\left(\alpha \int_{x}^{y} \phi_{k}^{\Delta}(v) \Delta v+(1-\alpha) \int_{x}^{y} \phi_{k}^{\nabla}(v) \nabla v\right) \lambda(y) \diamond_{\alpha} y .
\end{aligned}
$$

Then we can get the desired inequality (12) by

$$
\begin{aligned}
& \left|\prod_{k=1}^{m} \phi_{k}(x)-\frac{1}{m \int_{a}^{b} \lambda(y) \diamond_{\alpha} y} \sum_{k=1}^{m} \prod_{j \neq k} \phi_{j}(x) \int_{a}^{b} \phi_{k}(y) \lambda(y) \diamond_{\alpha} y\right| \\
= & \left|\frac{1}{m \int_{a}^{b} \lambda(y) \diamond_{\alpha} y} \sum_{k=1}^{m} \prod_{j \neq k} \phi_{j}(x) \int_{a}^{b}\left(\alpha \int_{x}^{y} \phi_{k}^{\Delta}(v) \Delta v+(1-\alpha) \int_{x}^{y} \phi_{k}^{\nabla}(v) \nabla v\right) \lambda(y) \diamond_{\alpha} y\right| \\
= & \frac{1}{m \int_{a}^{b} \lambda(y) \diamond_{\alpha} y} \sum_{k=1}^{m} \prod_{j \neq k}\left|\phi_{j}(x)\right| \int_{a}^{b}\left(\alpha \int_{x}^{y} \phi_{k}^{\Delta}(v) \Delta v+(1-\alpha) \int_{x}^{y} \phi_{k}^{\nabla}(v) \nabla v\right) \lambda(y) \diamond_{\alpha} y \mid \\
\leq & \frac{1}{m \int_{a}^{b} \lambda(y) \diamond_{\alpha} y} \sum_{k=1}^{m} \prod_{j \neq k}\left|\phi_{j}(x)\right| \int_{a}^{b}\left|\alpha \int_{x}^{y} \phi_{k}^{\Delta}(v) \Delta v+(1-\alpha) \int_{x}^{y} \phi_{k}^{\nabla}(v) \nabla v\right| \lambda(y) \diamond_{\alpha} y \\
\leq & \frac{1}{m \int_{a}^{b} \lambda(y) \diamond_{\alpha} y} \sum_{k=1}^{m} \prod_{j \neq k}\left|\phi_{j}(x)\right| \int_{a}^{b}\left(\alpha \int_{x}^{y}\left|\phi_{k}^{\Delta}(v)\right| \Delta v+(1-\alpha) \int_{x}^{y}\left|\phi_{k}^{\nabla}(v)\right| \nabla v\right) \lambda(y) \diamond_{\alpha} y \\
\leq & \frac{1}{m \int_{a}^{b} \lambda(y) \diamond_{\alpha} y} \sum_{k=1}^{m} \prod_{j \neq k}\left|\phi_{j}(x)\right| \int_{a}^{b}\left(\alpha \int_{x}^{y}\left\|\phi_{k}^{\Delta}\right\| \Delta v+(1-\alpha) \int_{x}^{y}\left\|\phi_{k}^{\nabla}\right\| \nabla v\right) \lambda(y) \diamond_{\alpha} y \\
\leq & \frac{1}{m \int_{a}^{b} \lambda(y) \diamond_{\alpha} y} \sum_{k=1}^{m}\left(\prod_{j \neq k}\left|\phi_{j}(x)\right|\left(\alpha\left\|\phi_{k}^{\Delta}\right\|\left\|_{\infty}+(1-\alpha)\right\| \phi_{k}^{\nabla} \|_{\infty}\right)\right) \int_{a}^{b} \lambda(y)|x-y| \diamond_{\alpha} y .
\end{aligned}
$$


From Inequality (14), we also have

$$
\begin{gathered}
m \int_{a}^{b} \lambda(y) \diamond_{\alpha} y \prod_{k=1}^{m} \phi_{k}(x) \lambda(x)-\lambda(x) \sum_{k=1}^{m} \prod_{j \neq k} \phi_{j}(x) \int_{a}^{b} \phi_{k}(y) \lambda(y) \diamond_{\alpha} y \\
=\lambda(x) \sum_{k=1}^{m} \prod_{j \neq k} \phi_{j}(x) \int_{a}^{b}\left(\alpha \int_{x}^{y} \phi_{k}^{\Delta}(v) \Delta v+(1-\alpha) \int_{x}^{y} \phi_{k}^{\nabla}(v) \nabla v\right) \lambda(y) \diamond_{\alpha} y .
\end{gathered}
$$

Diamond-Alpha integrating with respect to $x$, the inequality leads to

$$
\begin{aligned}
& \left|m \int_{a}^{b} \lambda(y) \diamond_{\alpha} y \int_{a}^{b} \prod_{k=1}^{m} \phi_{k}(x) \lambda(x) \diamond_{\alpha} x-\sum_{k=1}^{m}\left(\int_{a}^{b} \lambda(x) \prod_{j \neq k} \phi_{j}(x) \diamond_{\alpha} x \int_{a}^{b} \phi_{k}(x) \lambda(x) \diamond_{\alpha} x\right)\right| \\
= & \left|\sum_{k=1}^{m} \int_{a}^{b}\left(\lambda(x) \prod_{j \neq k} \phi_{j}(x) \int_{a}^{b}\left(\alpha \int_{x}^{y} \phi_{k}^{\Delta}(v) \Delta v+(1-\alpha) \int_{x}^{y} \phi_{k}^{\nabla}(v) \nabla v\right) \lambda(y) \diamond_{\alpha} y\right) \diamond_{\alpha} x\right| \\
\leq & \sum_{k=1}^{m} \int_{a}^{b}\left(\lambda(x)\left(\prod_{j \neq k}\left|\phi_{j}(x)\right|\left(\alpha\left\|\phi_{k}^{\Delta}\right\|_{\infty}+(1-\alpha)\left\|\phi_{k}^{\nabla}\right\|_{\infty}\right)\right) \int_{a}^{b} \lambda(y)|x-y| \diamond_{\alpha} y\right) \diamond_{\alpha} x \\
= & \int_{a}^{b} \lambda(x) \sum_{k=1}^{m}\left(\prod_{j \neq k} \phi_{j}(x)\left(\alpha\left\|\phi_{k}^{\Delta}\right\|_{\infty}+(1-\alpha)\left\|\phi_{k}^{\nabla}\right\|_{\infty}\right)\right)\left(\int_{a}^{b} \lambda(v)|x-y| \diamond_{\alpha} y\right) \diamond_{\alpha} x,
\end{aligned}
$$

which is the desired Inequality (13). Therefore, we complete the proof.

If we take $\mathbb{T}=\mathbb{R}$, then the inequalities in Theorem 4 change into

$$
\begin{aligned}
& \left|\prod_{k=1}^{m} \phi_{k}(x)-\frac{1}{m \int_{a}^{b} \lambda(y) \mathrm{d} y} \sum_{k=1}^{m}\left(\prod_{j \neq k} \phi_{j}(x) \int_{a}^{b} \lambda(y) \phi_{k}(y) \mathrm{d} y\right)\right| \\
\leq & \frac{1}{m \int_{a}^{b} \lambda(y) \mathrm{d} y} \sum_{k=1}^{m}\left(\prod_{j \neq k}\left|\phi_{j}(x)\right||| \phi_{k}^{\prime} \|_{\infty} \int_{a}^{b} \lambda(y)|x-y| \mathrm{d} y,\right.
\end{aligned}
$$

and

$$
\begin{aligned}
& \left|m \int_{a}^{b} \lambda(y) \phi_{k}(y) \mathrm{d} y \int_{a}^{b} \lambda(x) \prod_{k=1}^{m} \phi_{k}(x) \mathrm{d} x-\sum_{k=1}^{m}\left(\int_{a}^{b} \lambda(x) \prod_{j \neq k} \phi_{j}(x) \mathrm{d} x \int_{a}^{b} \lambda(x) \phi_{k}(x) \mathrm{d} x\right)\right| \\
\leq & \int_{a}^{b} \lambda(x) \sum_{k=1}^{m}\left(\prod_{j \neq k} \phi_{j}(x)\left\|\phi_{k}^{\prime}\right\|_{\infty}+\left(\int_{a}^{b} \lambda(v)|x-y| \mathrm{d} y\right) \mathrm{d} x .\right.
\end{aligned}
$$

In the same way, the inequalities in Theorem 2 are hold for the case with more functions. The theorem shows there is a upper bound of $\int_{a}^{b} \lambda(y) \prod_{k=1}^{n}\left(\phi_{k}(x)-\phi_{k}(y)\right) \diamond_{\alpha} y$ and the upper bound is depend on $\phi_{k}$ and $\lambda$.

Theorem 5. If $\lambda$ is a positive and continuous function, $\phi_{k}(k=1,2, \cdots, m) \in C\left([a, b]_{\mathbb{T}}, \mathbb{R}\right)$ are bounded, nonzero and satisfy

$$
\left\|\phi_{k}^{\Delta}\right\|_{\infty}=\sup _{x \in[a, b]}\left|\phi_{k}^{\Delta}(x)\right|<\infty, \quad\left\|\phi_{k}^{\nabla}\right\|_{\infty}=\sup _{x \in[a, b]}\left|\phi_{k}^{\nabla}(x)\right|<\infty, \quad k=1,2, \cdots, m,
$$

then the following inequality

$$
\left|\int_{a}^{b} \lambda(y) \prod_{k=1}^{n}\left(\phi_{k}(x)-\phi_{k}(y)\right) \diamond_{\alpha} y\right| \leq \prod_{k=1}^{m}\left(\alpha\left\|\phi_{k}^{\Delta}\right\|_{\infty}+(1-\alpha)\left\|\phi_{k}^{\nabla}\right\|_{\infty}\right) \int_{a}^{b} \lambda(y)|x-y|^{m} \diamond_{\alpha} y,
$$

holds for all $x \in[a, b]$. 
Proof. Clearly, we have

$$
\phi_{k}(y)-\phi_{k}(x)=\int_{x}^{y} \phi^{\Delta}(v) \Delta v=\int_{x}^{y} \phi^{\nabla}(v) \nabla v=\alpha \int_{x}^{y} \phi^{\Delta}(v) \Delta v+(1-\alpha) \int_{x}^{y} \phi^{\nabla}(v) \nabla v .
$$

Multiplying from $k=1$ to $m$, we obtain

$$
\prod_{k=1}^{m}\left(\phi_{k}(y)-\phi_{k}(x)\right)=\prod_{k=1}^{m}\left(\alpha \int_{x}^{y} \phi^{\Delta}(v) \Delta v+(1-\alpha) \int_{x}^{y} \phi^{\nabla}(v) \nabla v\right),
$$

namely,

$$
\lambda(y) \prod_{k=1}^{m}\left(\phi_{k}(y)-\phi_{k}(x)\right)=\lambda(y) \prod_{k=1}^{m}\left(\alpha \int_{x}^{y} \phi^{\Delta}(v) \Delta v+(1-\alpha) \int_{x}^{y} \phi^{\nabla}(v) \nabla v\right) .
$$

Diamond-Alpha integrating with respect to $y$ leads to

$$
\begin{aligned}
& \left|\int_{a}^{b} \lambda(y) \prod_{k=1}^{m}\left(\phi_{k}(y)-\phi_{k}(x)\right) \diamond_{\alpha} y\right| \\
= & \left|\int_{a}^{b} \lambda(y) \prod_{k=1}^{m}\left(\alpha \int_{x}^{y} \phi^{\Delta}(v) \Delta v+(1-\alpha) \int_{x}^{y} \phi^{\nabla}(v) \nabla v\right) \diamond_{\alpha} y\right| \\
\leq & \int_{a}^{b} \lambda(y) \prod_{k=1}^{m}\left|\alpha \int_{x}^{y} \phi^{\Delta}(v) \Delta v+(1-\alpha) \int_{x}^{y} \phi^{\nabla}(v) \nabla v\right| \diamond_{\alpha} y \\
\leq & \prod_{k=1}^{m}\left(\alpha\left\|\phi_{k}^{\Delta}\right\|_{\infty}+(1-\alpha)\left\|\phi_{k}^{\nabla}\right\|_{\infty}\right) \int_{a}^{b} \lambda(y)|x-y|^{m} \diamond_{\alpha} y,
\end{aligned}
$$

which is the desired Inequality (15).

If we chose $\mathbb{T}=\mathbb{R}$, then Inequality (15) transforms to

$$
\left|\int_{a}^{b} \lambda(y) \prod_{k=1}^{m}\left(\phi_{k}(y)-\phi_{k}(x)\right) \mathrm{d} y\right| \leq \prod_{k=1}^{m}\left\|\phi_{k}^{\prime}\right\|_{\infty} \int_{a}^{b} \lambda(y)|x-y|^{m} \mathrm{~d} y
$$

Next we consider the inequalities involving $m$ functions and $n$ variables based on the multiple Diamond- $\alpha_{i}$ integral in Section 2. We denote $D^{*}=\left[a_{1}, b_{1}\right] \times\left[a_{2}, b_{2}\right] \times \cdots\left[a_{n}, b_{n}\right]$.

Theorem 6. If $\lambda$ is a positive and continuous function with $n$ variables, $\phi_{k}(k=1,2, \cdots, m) \in$ $C\left(\left[a_{1}, b_{1}\right]_{\mathbb{T}_{1}} \times\left[a_{2}, b_{2}\right]_{\mathbb{T}_{2}} \times \cdots\left[a_{n}, b_{n}\right]_{\mathbb{T}_{n}}, \mathbb{R}\right)$ are bounded, nonzero and satisfy

$$
\left\|\phi_{k}^{\diamond \omega_{1} \diamond_{2} \cdots \omega_{\omega_{n}}}\right\|_{\infty}:=\sup _{\left(x_{1}, x_{2}, \cdots, x_{n}\right) \in D^{*}}\left|\frac{\partial^{n} \phi_{k}\left(x_{1}, x_{2}, \cdots, x_{n}\right)}{\diamond_{\omega_{1}} x_{1} \diamond \omega_{2} x_{2} \cdots \omega_{n} x_{n}}\right|<\infty, \quad k=1,2, \cdots, m,
$$

where $\omega_{i}=0$ or 1 for all $i=1,2, \cdots, n$ and $m \geq 2$ is given, then the following inequality holds for all $\left(x_{1}, x_{2}, \cdots, x_{n}\right) \in D^{*}$,

$$
\begin{aligned}
& \mid \prod_{k=1}^{m} \phi_{k}\left(x_{1}, x_{2}, \cdots, x_{n}\right)+\frac{1}{m M} \int_{a_{n}}^{b_{n}} \int_{a_{n-1}}^{b_{n-1}} \cdots \int_{a_{1}}^{b_{1}} \lambda\left(y_{1}, y_{2}, \cdots, y_{m}\right) \\
& \sum_{k=1}^{m}\left(\prod_{j \neq k} \phi_{j}\left(x_{1}, x_{2}, \cdots, x_{n}\right) \sum_{j=0}^{2^{n}-2} l_{n, j} I_{y}^{x}\left\{\phi_{k}\right\}(j)\right) \diamond_{\alpha_{1}} y_{1} \diamond_{\alpha_{2}} y_{2} \cdots \diamond_{\alpha_{n}} y_{n} \mid \\
\leq & \frac{1}{m M} \sum_{k=1}^{m}\left(\prod_{j \neq k}^{m}\left|\phi_{j}\left(x_{1}, x_{2}, \cdots, x_{n}\right)\right| \sum_{\omega_{1}, \cdots, \omega_{n}=0 \text { or } 1} \psi_{1}\left(\omega_{1}\right) \cdots \psi_{n}\left(\omega_{n}\right)\left\|\phi_{k}^{\diamond_{\omega_{1}} \diamond_{\omega_{2}} \cdots \diamond_{\omega_{n}}}\right\|_{\infty}\right. \\
& \int_{a_{n}}^{b_{n}} \int_{a_{n-1}}^{b_{n-1}} \cdots \int_{a_{1}}^{b_{1}} \lambda\left(y_{1}, y_{2}, \cdots, y_{m}\right) \prod_{j=1}^{n}\left|x_{i}-y_{i}\right| \diamond_{\alpha_{1}} y_{1} \diamond_{\alpha_{2}} y_{2} \cdots \diamond_{\alpha_{n}} y_{n},
\end{aligned}
$$


where

$$
M=\int_{a_{n}}^{b_{n}} \int_{a_{n-1}}^{b_{n-1}} \cdots \int_{a_{1}}^{b_{1}} \lambda\left(y_{1}, y_{2}, \cdots, y_{m}\right) \diamond_{\alpha_{1}} y_{1} \diamond_{\alpha_{2}} y_{2} \cdots \diamond_{\alpha_{n}} y_{n},
$$

and $l_{i, j}, I_{a}^{b}\{f\}(k)$ are defined by (6) and (7), respectively.

Proof. Same as (5), we have

$$
\begin{aligned}
& \int_{y_{n}}^{x_{n}} \int_{y_{n-1}}^{x_{n-1}} \cdots \int_{y_{1}}^{x_{1}} \frac{\partial^{n} \phi_{k}\left(v_{1}, v_{2}, \cdots, v_{n}\right)}{\Delta_{1} v_{1} \Delta_{2} v_{2} \cdots \Delta_{n} v_{n}} \Delta_{1} v_{1} \Delta_{2} v_{2} \cdots \Delta_{n} v_{n} \\
= & \sum_{j=0}^{2^{n}-1} l_{n, j} I_{y}^{x}\left\{\phi_{k}\right\}(j),
\end{aligned}
$$

for all $k=1,2,3, \cdots, m$. Moreover, it also holds for multiple mixed integral, namely,

$$
\begin{aligned}
& \int_{y_{n}}^{x_{n}} \int_{y_{n-1}}^{x_{n-1}} \cdots \int_{y_{1}}^{x_{1}} \frac{\partial^{n} \phi_{k}\left(v_{1}, v_{2}, \cdots, v_{n}\right)}{\diamond_{\omega_{1}} v_{1} \diamond_{\omega_{2}} v_{2} \cdots \diamond_{\omega_{n}} v_{n}} \diamond_{\omega_{1}} v_{1} \diamond_{\omega_{2}} v_{2} \cdots \diamond_{\omega_{n}} v_{n} \\
= & \sum_{j=0}^{2^{n}-1} l_{n, j} I_{y}^{x}\left\{\phi_{k}\right\}(j),
\end{aligned}
$$

where $\omega_{i}=1$ or 0 . Thus we can obtain

$$
\begin{aligned}
& \sum_{j=0}^{2^{n}-1} l_{n, j} I_{y}^{x}\left\{\phi_{k}\right\}(j) \\
= & \sum_{\omega_{1}, \cdots, \omega_{n}=0 \text { or } 1} \psi_{1}\left(\omega_{1}\right) \cdots \psi_{n}\left(\omega_{n}\right) \\
& \int_{y_{n}}^{x_{n}} \int_{y_{n-1}}^{x_{n-1}} \cdots \int_{y_{1}}^{x_{1}} \frac{\partial^{n} \phi_{k}\left(v_{1}, v_{2}, \cdots, v_{n}\right)}{\omega_{1} v_{1} \diamond_{\omega_{2}} v_{2} \cdots \diamond_{\omega_{n}} v_{n}} \diamond_{\omega_{1}} v_{1} \diamond_{\omega_{2}} v_{2} \cdots \diamond_{\omega_{n}} v_{n},
\end{aligned}
$$

where $\psi_{k}$ are defined by (9). Adding up from $k=1$ to $m$ and multiplying

$$
\lambda\left(y_{1}, y_{2}, \cdots, y_{m}\right) \prod_{j=1, j \neq k}^{m} \phi_{j}\left(x_{1}, x_{2}, \cdots, x_{n}\right),
$$

Inequality (17) leads to

$$
\begin{aligned}
& \lambda\left(y_{1}, y_{2}, \cdots, y_{m}\right) \sum_{k=1}^{m}\left(\prod_{j \neq k} \phi_{j}\left(x_{1}, x_{2}, \cdots, x_{n}\right) \sum_{j=0}^{2^{n}-1} l_{n, j} I_{y}^{x}\left\{\phi_{k}\right\}(j)\right) \\
= & \lambda\left(y_{1}, y_{2}, \cdots, y_{m}\right) \sum_{k=1}^{m}\left(\prod _ { j \neq k } \phi _ { j } ( x _ { 1 } , x _ { 2 } , \cdots , x _ { n } ) \left(\phi_{k}\left(x_{1}, x_{2}, \cdots, x_{n}\right)\right.\right. \\
& \left.\left.+\sum_{j=0}^{2^{n}-2} l_{n, j} I_{y}^{x}\left\{\phi_{k}\right\}(j)\right)\right) \\
= & m \lambda\left(y_{1}, y_{2}, \cdots, y_{m}\right) \prod_{k=1}^{m} \phi_{k}\left(x_{1}, x_{2}, \cdots, x_{n}\right) \\
& +\lambda\left(y_{1}, y_{2}, \cdots, y_{m}\right) \sum_{k=1}^{m}\left(\prod_{j \neq k} \phi_{j}\left(x_{1}, x_{2}, \cdots, x_{n}\right) \sum_{j=0}^{2^{n}-2} l_{n, j} I_{y}^{x}\left\{\phi_{k}\right\}(j)\right) \\
= & \lambda\left(y_{1}, y_{2}, \cdots, y_{m}\right) \sum_{k=1}^{m}\left(\prod_{j \neq k} \phi_{j}\left(x_{1}, x_{2}, \cdots, x_{n}\right) \sum_{\omega_{1}, \cdots, \omega_{n}=0 \text { or } 1} \psi_{1}\left(\omega_{1}\right) \cdots \psi_{n}\left(\omega_{n}\right)\right. \\
& \left.\int_{y_{n}}^{x_{n}} \int_{y_{n-1}}^{x_{n-1}} \cdots \int_{y_{1}}^{x_{1}} \frac{\partial^{n} \phi_{k}\left(v_{1}, v_{2}, \cdots, v_{n}\right)}{\omega_{1} v_{\omega_{2}} v_{2} \cdots \diamond_{\omega_{n}} v_{n}} \diamond_{\omega_{1}} v_{1} \diamond_{\omega_{2}} v_{2} \cdots \diamond_{\omega_{n}} v_{n}\right) .
\end{aligned}
$$


Then we can get the desired inequality by taking multiple Diamond- $\alpha_{i}$ integral with respect to $x_{i}$,

$$
\begin{aligned}
& \mid \prod_{k=1}^{m} \phi_{k}\left(x_{1}, x_{2}, \cdots, x_{n}\right)+\frac{1}{m M} \int_{a_{n}}^{b_{n}} \int_{a_{n-1}}^{b_{n-1}} \cdots \int_{a_{1}}^{b_{1}} \lambda\left(y_{1}, y_{2}, \cdots, y_{m}\right) \\
& \sum_{k=1}^{m}\left(\prod_{j \neq k} \phi_{j}\left(x_{1}, x_{2}, \cdots, x_{n}\right) \sum_{j=0}^{2^{n}-2} l_{n, j} I_{y}^{x}\left\{\phi_{k}\right\}(j)\right) \diamond_{\alpha_{1}} y_{1} \diamond_{\alpha_{2}} y_{2} \cdots \diamond_{\alpha_{n}} y_{n} \mid \\
& =\frac{1}{m M} \mid \int_{a_{n}}^{b_{n}} \cdots \int_{a_{1}}^{b_{1}} \lambda\left(y_{1}, \cdots, y_{m}\right) \sum_{k=1}^{m}\left(\prod_{j \neq k} \phi_{j}\left(x_{1}, \cdots, x_{n}\right) \sum_{\omega_{1}, \cdots, \omega_{n}=0 \text { or } 1} \psi_{1}\left(\omega_{1}\right) \cdots \psi_{n}\left(\omega_{n}\right)\right. \\
& \left.\int_{y_{n}}^{x_{n}} \cdots \int_{y_{1}}^{x_{1}} \frac{\partial^{n} \phi_{k}\left(v_{1}, \cdots, v_{n}\right)}{\diamond_{\omega_{1}} v_{1} \cdots \diamond_{\omega_{n}} v_{n}} \diamond_{\omega_{1}} v_{1} \cdots \diamond_{\omega_{n}} v_{n}\right) \diamond_{\alpha_{1}} y_{1} \cdots \diamond_{\alpha_{n}} y_{n} \mid \\
& \leq \frac{1}{m M} \int_{a_{n}}^{b_{n}} \cdots \int_{a_{1}}^{b_{1}} \lambda\left(y_{1}, \cdots, y_{m}\right) \sum_{k=1}^{m}\left(\prod_{j \neq k}\left|\phi_{j}\left(x_{1}, \cdots, x_{n}\right)\right| \sum_{\omega_{1}, \cdots, \omega_{n}=0 \text { or } 1} \psi_{1}\left(\omega_{1}\right) \cdots \psi_{n}\left(\omega_{n}\right)\right. \\
& \left.\left|\int_{y_{n}}^{x_{n}} \cdots \int_{y_{1}}^{x_{1}} \frac{\partial^{n} \phi_{k}\left(v_{1}, \cdots, v_{n}\right)}{\diamond_{\omega_{1}} v_{1} \cdots \diamond_{\omega_{n}} v_{n}} \diamond_{\omega_{1}} v_{1} \cdots \diamond_{\omega_{n}} v_{n}\right|\right) \diamond_{\alpha_{1}} y_{1} \cdots \diamond_{\alpha_{n}} y_{n} \\
& \leq \frac{1}{m M} \sum_{k=1}^{m}\left(\prod_{j \neq k}\left|\phi_{j}\left(x_{1}, x_{2}, \cdots, x_{n}\right)\right| \sum_{\omega_{1}, \cdots, \omega_{n}=0 \text { or } 1} \psi_{1}\left(\omega_{1}\right) \cdots \psi_{n}\left(\omega_{n}\right)\left\|\phi_{k}^{\diamond \omega_{1} \diamond \omega_{2} \cdots \omega_{n}}\right\|_{\infty}\right. \\
& \int_{a_{n}}^{b_{n}} \int_{a_{n-1}}^{b_{n-1}} \cdots \int_{a_{1}}^{b_{1}} \lambda\left(y_{1}, y_{2}, \cdots, y_{m}\right) \prod_{j=1}^{n}\left|x_{i}-y_{i}\right| \diamond_{\alpha_{1}} y_{1} \diamond_{\alpha_{2}} y_{2} \cdots \diamond_{\alpha_{n}} y_{n} .
\end{aligned}
$$

Remark 2. It is clear that Inequality (16) changes into (12) by taking $n=1$ in Theorem 6 .

If $n=2$, then Theorem 6 gives the two dimensional Diamond-Alpha Ostrowski type inequalities.

Corollary 4. If $\lambda$ is a positive and continuous function of two variables, $\phi_{k}(k=1,2, \cdots, m) \in$ $C\left(\left[a_{1}, b_{1}\right]_{\mathbb{T}_{1}} \times\left[a_{2}, b_{2}\right]_{\mathbb{T}_{2}}, \mathbb{R}\right)$ are bounded, nonzero and satisfy

$$
\begin{aligned}
& \left\|\phi_{k}^{\Delta_{1} \Delta_{2}}\right\|_{\infty}:=\sup _{\left(x_{1}, x_{2}\right) \in D_{2}^{*}}\left|\frac{\partial^{2} \phi_{k}\left(x_{1}, x_{2}\right)}{\Delta_{1} x_{1} \Delta_{2} x_{2}}\right|<\infty, \quad\left\|\phi_{k}^{\Delta_{1} \nabla_{2}}\right\|_{\infty}:=\sup _{\left(x_{1}, x_{2}\right) \in D_{2}^{*}}\left|\frac{\partial^{2} \phi_{k}\left(x_{1}, x_{2}\right)}{\Delta_{1} x_{1} \nabla_{2} x_{2}}\right|<\infty \\
& \left\|\phi_{k}^{\nabla_{1} \Delta_{2}}\right\|_{\infty}:=\sup _{\left(x_{1}, x_{2}\right) \in D_{2}^{*}}\left|\frac{\partial^{2} \phi_{k}\left(x_{1}, x_{2}\right)}{\nabla_{1} x_{1} \Delta_{2} x_{2}}\right|<\infty, \quad\left\|\phi_{k}^{\nabla_{1} \nabla_{2}}\right\|_{\infty}:=\sup _{\left(x_{1}, x_{2}\right) \in D_{2}^{*}}\left|\frac{\partial^{2} \phi_{k}\left(x_{1}, x_{2}\right)}{\nabla_{1} x_{1} \nabla_{2} x_{2}}\right|<\infty
\end{aligned}
$$

then we have

$$
\begin{aligned}
& \mid \prod_{k=1}^{m} \phi_{k}\left(x_{1}, x_{2}\right)+\frac{1}{m M} \int_{a_{2}}^{b_{2}} \int_{a_{1}}^{b_{1}} \lambda\left(y_{1}, y_{2}\right) \\
& \sum_{k=1}^{m}\left(\prod_{j \neq k} \phi_{j}\left(x_{1}, x_{2}\right)\left(-\phi_{k}\left(x_{1}, y_{2}\right)-\phi_{k}\left(y_{1}, x_{2}\right)+\phi_{k}\left(y_{1}, y_{2}\right)\right)\right) \diamond_{\alpha_{1}} y_{1} \diamond_{\alpha_{2}} y_{2} \mid \\
\leq & \frac{1}{m M} \sum_{k=1}^{m}\left(\prod_{j \neq k}\left|\phi_{j}\left(x_{1}, x_{2}\right)\right| \sum_{\omega_{1}, \omega_{2}=0 \text { or } 1} \psi_{1}\left(\omega_{1}\right) \psi_{2}\left(\omega_{2}\right)\left\|\phi_{k}^{\diamond \omega_{1} \diamond \omega_{2}}\right\|_{\infty}\right. \\
& \int_{a_{2}}^{b_{2}} \int_{a_{1}}^{b_{1}} \lambda\left(y_{1}, y_{2}\right)\left|\left(x_{1}-y_{1}\right)\left(x_{2}-y_{2}\right)\right| \diamond_{\alpha_{1}} y_{1} \diamond_{\alpha_{2}} y_{2},
\end{aligned}
$$


for all $\left(x_{1}, x_{2}\right) \in\left[a_{1}, b_{1}\right] \times\left[a_{2}, b_{2}\right]$, where $D_{2}^{*}=\left[a_{1}, b_{1}\right] \times\left[a_{2}, b_{2}\right]$ and

$$
M=\int_{a_{2}}^{b_{2}} \int_{a_{1}}^{b_{1}} \lambda\left(y_{1}, y_{2}\right) \diamond_{\alpha_{1}} y_{1} \diamond_{\alpha_{2}} y_{2} .
$$

Since $\diamond_{1}=\Delta$ and $\diamond_{0}=\nabla$, then the corollary above gives the following corollaries when $\alpha_{1}=\alpha_{2}=\cdots=\alpha_{n}=0$ or 1 .

Corollary 5. If the conditions in Theorem 6 hold, then we have

$$
\begin{aligned}
& \mid \prod_{k=1}^{m} \phi_{k}\left(x_{1}, x_{2}, \cdots, x_{n}\right)+\frac{1}{m M} \int_{a_{n}}^{b_{n}} \int_{a_{n-1}}^{b_{n-1}} \cdots \int_{a_{1}}^{b_{1}} \lambda\left(y_{1}, y_{2}, \cdots, y_{m}\right) \\
& \sum_{k=1}^{m}\left(\prod_{j \neq k} \phi_{j}\left(x_{1}, x_{2}, \cdots, x_{n}\right) \sum_{j=0}^{2^{n}-2} l_{n, j} j_{y}^{x}\left\{\phi_{k}\right\}(j)\right) \Delta_{1} y_{1} \Delta_{2} y_{2} \cdots \Delta_{n} y_{n} \mid \\
\leq & \frac{1}{m M} \sum_{k=1}^{m}\left(\prod_{j \neq k}\left|\phi_{j}\left(x_{1}, x_{2}, \cdots, x_{n}\right)\right| \sum_{\omega_{1}, \cdots, \omega_{n}=0 \text { or } 1} \psi_{1}\left(\omega_{1}\right) \cdots \psi_{n}\left(\omega_{n}\right)\left\|\phi_{k}^{\diamond \omega_{1} \diamond_{\omega_{2}} \cdots \diamond_{\omega_{n}}}\right\|_{\infty}\right. \\
& \int_{a_{n}}^{b_{n}} \int_{a_{n-1}}^{b_{n-1}} \cdots \int_{a_{1}}^{b_{1}} \lambda\left(y_{1}, y_{2}, \cdots, y_{m}\right) \prod_{j=1}^{n}\left|x_{i}-y_{i}\right| \Delta_{1} y_{1} \Delta_{2} y_{2} \cdots \Delta_{n} y_{n},
\end{aligned}
$$

for all $\left(x_{1}, x_{2}, \cdots, x_{n}\right) \in D$, where

$$
M=\int_{a_{n}}^{b_{n}} \int_{a_{n-1}}^{b_{n-1}} \cdots \int_{a_{1}}^{b_{1}} \lambda\left(y_{1}, y_{2}, \cdots, y_{m}\right) \Delta_{1} y_{1} \Delta_{2} y_{2} \cdots \Delta_{n} y_{n} .
$$

Corollary 6. If the conditions in Theorem 6 hold, then we have

$$
\begin{aligned}
& \mid \prod_{k=1}^{m} \phi_{k}\left(x_{1}, x_{2}, \cdots, x_{n}\right)+\frac{1}{m M} \int_{a_{n}}^{b_{n}} \int_{a_{n-1}}^{b_{n-1}} \cdots \int_{a_{1}}^{b_{1}} \lambda\left(y_{1}, y_{2}, \cdots, y_{m}\right) \\
& \sum_{k=1}^{m}\left(\prod_{j \neq k} \phi_{j}\left(x_{1}, x_{2}, \cdots, x_{n}\right) \sum_{j=0}^{2^{n}-2} l_{n, j} I_{y}^{x}\left\{\phi_{k}\right\}(j)\right) \nabla_{1} y_{1} \nabla_{2} y_{2} \cdots \nabla_{n} y_{n} \mid \\
\leq & \frac{1}{m M} \sum_{k=1}^{m}\left(\prod_{j \neq k}\left|\phi_{j}\left(x_{1}, x_{2}, \cdots, x_{n}\right)\right| \sum_{\omega_{1}, \cdots, \omega_{n}=0 \text { or } 1} \psi_{1}\left(\omega_{1}\right) \cdots \psi_{n}\left(\omega_{n}\right)\left\|\phi_{k}^{\diamond_{\omega_{1}} \diamond_{\omega_{2}} \cdots \diamond_{\omega_{n}}}\right\|_{\infty}\right. \\
& \int_{a_{n}}^{b_{n}} \int_{a_{n-1}}^{b_{n-1}} \cdots \int_{a_{1}}^{b_{1}} \lambda\left(y_{1}, y_{2}, \cdots, y_{m}\right) \prod_{j=1}^{n}\left|x_{i}-y_{i}\right| \nabla_{1} y_{1} \nabla_{2} y_{2} \cdots \nabla_{n} y_{n},
\end{aligned}
$$

for all $\left(x_{1}, x_{2}, \cdots, x_{n}\right) \in D$, where

$$
M=\int_{a_{n}}^{b_{n}} \int_{a_{n-1}}^{b_{n-1}} \cdots \int_{a_{1}}^{b_{1}} \lambda\left(y_{1}, y_{2}, \cdots, y_{m}\right) \nabla_{1} y_{1} \nabla_{2} y_{2} \cdots \nabla_{n} y_{n} .
$$

\section{Examples}

In this section, we provide some examples to further illustrate our inequalities. Examples 2 and 3 are examples of Theorem 4 and Example 4 is an example of Theorem 6. In fact, there are many interesting inequalities that can be derived from Theorems 4-6 and the following three examples provide only a few special cases. 
Example 2. Consider the inequality:

$$
\begin{aligned}
& \left|x^{\frac{m(m+1)}{2}}-\frac{2}{m\left(b^{2}-a^{2}\right)} \sum_{k=1}^{m}\left(\frac{b^{k+2}-a^{k+2}}{k+2} x^{\frac{m(m+1)}{2}-k}\right)\right| \\
\leq & \frac{2}{m\left(b^{2}-a^{2}\right)} \sum_{k=1}^{m}\left(k b^{k-1} x^{\frac{m(m+1)}{2}-k}\right) \int_{a}^{b} y|x-y| d y, \quad 0<a \leq x \leq b .
\end{aligned}
$$

Proof. Taking $\mathbb{T}=\mathbb{R}, \lambda(y)=y, \phi_{k}(x)=x^{k}$ in Theorem 4 , we have

$$
\left\|\phi_{k}^{\Delta}\right\|_{\infty}=\left\|\phi_{k}^{\nabla}\right\|_{\infty}=\left\|\phi_{k}^{\prime}\right\|_{\infty}=k b^{k-1},
$$

and

$$
\int_{a}^{b} \lambda(y) \diamond_{\alpha} y=\int_{a}^{b} y \mathrm{~d} y=\frac{b^{2}-a^{2}}{2}, \quad \int_{a}^{b} \lambda(y) \phi_{k}(y) \diamond_{\alpha} y=\int_{a}^{b} y^{k+1} \mathrm{~d} y=\frac{b^{k+2}-a^{k+2}}{k+2} .
$$

Hence we can get the desired Inequality (19) according to (12).

Example 3. Consider the inequality:

$$
\begin{aligned}
& \left|\frac{b^{k+2}-a^{k+2}}{k+2}\left(\frac{4}{11}\left(b^{11}-a^{11}\right)-\sum_{k=1}^{4} \frac{b^{12-k}-a^{12-k}}{12-k}\right)\right| \\
\leq & \int_{a}^{b} k b^{k-1} \sum_{k=1}^{4} x^{11-k}\left(\int_{a}^{b} y|x-y| d y\right) d x, \quad 0<a \leq x \leq b .
\end{aligned}
$$

Proof. Taking $\mathbb{T}=\mathbb{R}, \lambda(y)=y, \phi_{k}(x)=x^{k}, m=4$ in Theorem 4 , we can get the desired Inequality (20) according to (13).

Example 4. Consider the inequality:

$$
\begin{gathered}
\mid \begin{array}{l}
x_{1}^{3} x_{2}^{3}-P_{1}(N)\left(x_{1}^{3} x_{2}^{2}+x_{1}^{2} x_{2}^{3}\right)+P_{2}(N) x_{1}^{2} x_{2}^{2}-P_{3}(N)\left(x_{1}^{3} x_{2}+x_{1} x_{2}^{3}\right)+P_{4}(N) x_{1} x_{2} \mid \\
\leq \frac{1}{2 M}\left(x_{1}^{2} x_{2}^{2}+4 N^{2} x_{1} x_{2}\right) \int_{a_{2}}^{b_{2}} \int_{a_{1}}^{b_{1}} y_{1} y_{2}\left|\left(x_{1}-y_{1}\right)\left(x_{2}-y_{2}\right)\right| \diamond_{\frac{1}{2}} y_{1} \diamond_{\frac{1}{2}} y_{2}, \quad\left(x_{1}, x_{2}\right) \in[1, N]_{\mathbb{N}}^{2} \\
\text { where } N \geq 3, N \in \mathbb{N}, M=f_{0}(N) \text { and } \\
P_{0}(N)=\frac{N^{4}+2 N^{3}-3 N^{2}-8 N-4}{16}, \quad P_{1}(N)=\frac{2 N^{5}+5 N^{4}-8 N^{3}-11 N^{2}-12 N-12}{96 P_{0}(N)}, \\
P_{2}(N)=\frac{4 N^{6}+12 N^{5}+7 N^{4}+6 N^{3}-11 N^{2}-6}{288 P_{0}(N)}, \quad P_{3}(N)=\frac{N^{6}+3 N^{5}-5 N^{4}-7 N^{3}-8 N-8}{64 P_{0}(N)}, \\
P_{4}(N)=\frac{N^{8}+4 N^{7}+-10 N^{6}+4 N^{5}+N^{4}-32 N^{3}-16}{128 P_{0}(N)} .
\end{array}
\end{gathered}
$$

Proof. Taking $\mathbb{T}=\mathbb{N}, n=m=2, \phi_{k}\left(x_{1}, x_{2}\right)=x_{1}^{k} x_{2}^{k}, \lambda\left(y_{1}, y_{2}\right)=y_{1} y_{2}, a_{1}=a_{2}=1, b_{1}=$ $b_{2}=N$ and $\alpha_{1}=\alpha_{2}=\frac{1}{2}$ in Theorem 6 , the left-hand side of Inequality (18) transforms into 


$$
\begin{aligned}
& \mid \prod_{k=1}^{m} \phi_{k}\left(x_{1}, x_{2}\right)+\frac{1}{m M} \int_{a_{2}}^{b_{2}} \int_{a_{1}}^{b_{1}} \lambda\left(y_{1}, y_{2}\right) \\
& \sum_{k=1}^{m}\left(\prod_{j \neq k} \phi_{j}\left(x_{1}, x_{2}\right)\left(-\phi_{k}\left(x_{1}, y_{2}\right)-\phi_{k}\left(y_{1}, x_{2}\right)+\phi_{k}\left(y_{1}, y_{2}\right)\right)\right) \diamond_{\alpha_{1}} y_{1} \diamond_{\alpha_{2}} y_{2} \mid \\
= & \left|x_{1}^{3} x_{2}^{3}+\frac{1}{2 M} \int_{1}^{N} \int_{1}^{N} y_{1} y_{2} \sum_{k=1}^{2}\left(x_{1}^{3-k} x_{2}^{3-k}\left(-x_{1}^{k} y_{2}^{k}-x_{2}^{k} y_{1}^{k}+y_{1}^{k} y_{2}^{k}\right)\right) \diamond_{\alpha_{1}} y_{1} \diamond_{\alpha_{2}} y_{2}\right| \\
= & \mid x_{1}^{3} x_{2}^{3}+\frac{1}{2 M} \int_{1}^{N} \int_{1}^{N} \sum_{k=1}^{2}\left(-x_{1}^{3} x_{2}^{3-k} y_{1} y_{2}^{k+1}-x_{1}^{3-k} x_{2}^{3} y_{1}^{k+1} y_{2}\right. \\
& \left.+x_{1}^{3-k} x_{2}^{3-k} y_{1}^{k+1} y_{2}^{k+1}\right) \diamond_{\alpha_{1}} y_{1} \diamond_{\alpha_{2}} y_{2} \mid \\
= & \mid x_{1}^{3} x_{2}^{3}+\frac{1}{2 M} \int_{1}^{N} \int_{1}^{N}-x_{1}^{3} x_{2}^{2} y_{1} y_{2}^{2}-x_{1}^{2} x_{2}^{3} y_{1}^{2} y_{2}+x_{1}^{2} x_{2}^{2} y_{1}^{2} y_{2}^{2} \\
& -x_{1}^{3} x_{2} y_{1} y_{2}^{3}-x_{1} x_{2}^{3} y_{1}^{3} y_{2}+x_{1} x_{2} y_{1}^{3} y_{2}^{3} \diamond_{\alpha_{1}} y_{1} \diamond_{\alpha_{2}} y_{2} \mid \\
= & \mid x_{1}^{3} x_{2}^{3}+\frac{1}{2 M}\left(\sum_{\omega_{1}, \omega_{2}=0} \psi_{1}\left(\omega_{1}\right) \psi_{2}\left(\omega_{2}\right) \int_{1}^{N} \int_{1}^{N}-x_{1}^{3} x_{2}^{2} y_{1} y_{2}^{2}-x_{1}^{2} x_{2}^{3} y_{1}^{2} y_{2}+x_{1}^{2} x_{2}^{2} y_{1}^{2} y_{2}^{2}\right. \\
& -x_{1}^{3} x_{2} y_{1} y_{2}^{3}-x_{1} x_{2}^{3} y_{1}^{3} y_{2}+x_{1} x_{2} y_{1}^{3} y_{2}^{3} \diamond_{\omega_{1}} y_{1} \diamond_{\omega_{2}} y_{2} \mid \\
= & \mid x_{1}^{3} x_{2}^{3}-\frac{1}{2 M}\left(x_{1}^{3} x_{2}^{2}+x_{1}^{2} x_{2}^{3}\right) \frac{N(N+1) N(N+1)(2 N+1)-12\left(N^{3}+N^{2}+N+1\right)}{4 N_{1}} \\
& +\frac{1}{2 M} x_{1}^{2} x_{2}^{2} \frac{N^{2}(N+1)^{2}(2 N+1)^{2}-6\left(N^{4}+2 N^{2}+1\right)}{24} \\
& -\frac{1}{2 M}\left(x_{1}^{3} x_{2}+x_{1} x_{2}^{3}\right) \frac{N^{3}(N+1)^{3}-8\left(N^{4}+N^{3}+N+1\right)}{32} \\
& +\frac{1}{2 M} x_{1} x_{2} \frac{N^{4}(N+1)^{4}-16\left(N^{6}+2 N^{3}+1\right)}{64} \mid \\
= & x_{1}^{3} x_{2}^{3}-P_{1}(N)\left(x_{1}^{3} x_{2}^{2}+x_{1}^{2} x_{2}^{3}\right)+P_{2}(N) x_{1}^{2} x_{2}^{2}-P_{3}(N)\left(x_{1}^{3} x_{2}+x_{1} x_{2}^{3}\right)+P_{4}(N) x_{1} x_{2} \mid .
\end{aligned}
$$

The right-hand side of Inequality (18) transforms into

$$
\begin{aligned}
& \frac{1}{m M} \sum_{k=1}^{m}\left(\prod_{j \neq k}\left|\phi_{j}\left(x_{1}, x_{2}\right)\right| \sum_{\omega_{1}, \omega_{2}=0 \text { or } 1} \psi_{1}\left(\omega_{1}\right) \psi_{2}\left(\omega_{2}\right)\left\|\phi_{k}^{\diamond \omega_{1} \diamond_{\omega_{2}}}\right\|_{\infty}\right. \\
& \int_{a_{2}}^{b_{2}} \int_{a_{1}}^{b_{1}} \lambda\left(y_{1}, y_{2}\right)\left|\left(x_{1}-y_{1}\right)\left(x_{2}-y_{2}\right)\right| \diamond_{\alpha_{1}} y_{1} \diamond_{\alpha_{2}} y_{2} \\
= & \frac{1}{2 M} \sum_{k=1}^{2}\left(x_{1}^{3-k} x_{2}^{3-k} \frac{1}{4}\left(\left\|\phi_{k}^{\Delta_{1} \Delta_{2}}\right\|_{\infty}+\left\|\phi_{k}^{\Delta_{1} \nabla_{2}}\right\|_{\infty}+\left\|\phi_{k}^{\nabla_{1} \Delta_{2}}\right\|_{\infty}+\left\|\phi_{k}^{\nabla_{1} \nabla_{2}}\right\|_{\infty}\right)\right. \\
& \int_{a_{2}}^{b_{2}} \int_{a_{1}}^{b_{1}} \lambda\left(y_{1}, y_{2}\right)\left|\left(x_{1}-y_{1}\right)\left(x_{2}-y_{2}\right)\right| \diamond_{\alpha_{1}} y_{1} \diamond_{\alpha_{2}} y_{2} \\
= & \frac{1}{2 M} \sum_{k=1}^{2}\left(\frac { x _ { 1 } ^ { 3 - k } x _ { 2 } ^ { 3 - k } } { 4 } \left(\left((N+1)^{k}-N^{k}\right)\left((N+1)^{k}-N^{k}\right)\right.\right. \\
& +\left((N+1)^{k}-N^{k}\right)\left(N^{k}-(N-1)^{k}\right)+\left(N^{k}-(N-1)^{k}\right)\left((N+1)^{k}-N^{k}\right) \\
& \left.+\left(N^{k}-(N-1)^{k}\right)\left(N^{k}-(N-1)^{k}\right)\right) \int_{a_{2}}^{b_{2}} \int_{a_{1}}^{b_{1}} y_{1} y_{2}\left|\left(x_{1}-y_{1}\right)\left(x_{2}-y_{2}\right)\right| \diamond_{\alpha_{1}} y_{1} \diamond_{\alpha_{2}} y_{2} \\
= & \frac{1}{2 M}\left(x_{1}^{2} x_{2}^{2}+4 N^{2} x_{1} x_{2}\right) \int_{a_{2}}^{b_{2}} \int_{a_{1}}^{b_{1}} y_{1} y_{2}\left|\left(x_{1}-y_{1}\right)\left(x_{2}-y_{2}\right)\right| \diamond_{\frac{1}{2}} y_{1} \diamond_{\frac{1}{2}} y_{2} .
\end{aligned}
$$


We can complete the proof by combining Inequalities (21) and (22).

\section{Conclusions}

In this paper, we give the definition of multiple Diamond-Alpha integral with different $\alpha$. Some Ostrowski type inequalities with more functions and higher dimensions are established.

The new integral has wide applications; it can represent multiple mixed integrals and generalize inequalities effectively. Moreover, it will play a key role in the process of researching Diamond-Alpha dynamic equations, which is also the aim in the following work.

Author Contributions: Conceptualization, Z.-X.M.; methodology, Z.-X.M. and J.-P.H.; formal analysis, Z.-X.M., J.-P.H., C.-P.M. and S.-P.L.; writing—original draft preparation, Z.-X.M.; writing—review and editing, Z.-X.M. and Y.-R.Z.; supervision, Y.-R.Z.; funding acquisition, Y.-R.Z. All authors have read and agreed to the published version of the manuscript.

Funding: This research was funded by the Fundamental Research Funds for the Central Universities under Grant MS117.

Institutional Review Board Statement: Not applicable.

Informed Consent Statement: Not applicable.

Data Availability Statement: All data generated or analyzed during this study are included in this published article.

Acknowledgments: The authors would like to express their sincere thanks to the anonymous referees for their great efforts to improve this paper.

Conflicts of Interest: The authors declare no conflict of interest.

\section{References}

1. Hilger, S. Ein Maßkettenkalkül mit Anwendung auf Zentrumsmannigfaltigkeiten. Ph.D. Thesis, Universität Würzburg, Würzburg, Germany, 1988.

2. Bohner, M.; Georgiev, S.G. Multivariable Dynamic Calculus on Time Scales; Spriner: Cham, Switzerland, 2016. [CrossRef]

3. Hu, X.-M.; Tian, J.-F.; Chu, Y.-M. On Cauchy-Schwarz inequality for N-tuple Diamond-Alpha integral. J. Inequal. Appl. 2020, 2020, 1-15.

4. Bohner, M.; Guseinov, G.S.H. Multiple integration on time scales. Dynam. Syst. Appl. 2005, 14, 449-515.

5. Ferreira, R.A.C.; Torres, D.F.M. Remarks on the calculus of variations on time scales. Ecol. Econ. 2007, 9, 65-73.

6. Bohner, M.; Guseinov, G.S. Multiple lebesgue integration on time scals. Adv. Differ. Equ. 2006, 1, 1-12. [CrossRef]

7. Sun, Y.; Hassan, T. Some nonlinear dynamic integral inequalities on time scales. Appl. Math. Comput. 2013, 220, 221-225. [CrossRef]

8. Bohner, M.; Guseinov, G.S. Double integral calculus of variations on time scales. Comput. Math. Appl. 2006, 54, 45-57. [CrossRef]

9. Hussain, S.; Latif, M.A.; Alomari, M. Generalized double-integral Ostrowski type inequalities on time scales. Appl. Math. Lett. 2011, 24, 1461-1467. [CrossRef]

10. Seiffertt, J.; Sanyal, S.; Wunsch, D.C. Hamilton-Jacobi-Bellman equations and approximate dynamic programming on time scales. IEEE Trans. Syst. Man. Cybern. B. 2008, 38, 918-923. [CrossRef]

11. Bohner, M.; Guseinov, G.S. Line integrals and Green's formula on time scales. J. Math. Anal. Appl. 2007, 326, 1124-1141. [CrossRef]

12. Tian, J.-F.; Ha, M.-H. Extensions of Hölder-type inequalities on time scales and their applications. J. Nonlinear Sci. Appl. 2017, 10, 937-953. [CrossRef]

13. Mao, Z.-X.; Zhu, Y.-R.; Guo, B.-H. Qi type diamond-alpha integral inequalities. Mathematics 2021, 9, 449. [CrossRef]

14. Zhang, Z.-Y.; Feng, R.-H.; Jadlovská, I.; Liu, Q. Oscillation criteria for third-order nonlinear neutral dynamic equations with mixed deviating arguments on time scales. Mathematics 2021, 9, 552. [CrossRef]

15. Saker, S.; Kenawy, M.; AlNemer, G.; Zakarya, M. Some fractional dynamic inequalities of Hardy's type via conformable calculus. Mathematics 2020, 8, 434. [CrossRef]

16. Sheng, Q.; Fadag, M.; Henderson, J.; Davis, J.M. An exploration of combined dynamic derivatives on time scales and their applications. Nonlinear Anal-Real. 2006, 7, 395-413. [CrossRef] [PubMed]

17. Tian, J.-F. Triple Diamond-Alpha integral and Hölder-type inequalities. J. Inequal. Appl. 2018, 2018, 1-14. [CrossRef]

18. Rogers, J.W.; Sheng, Q. Note on the diamond- $\alpha$ dymanic derivative on time scales. J. Math. Anal. Appl. 2007, 326, 228-241. [CrossRef]

19. Özkan, U.M.; Kaymakçalan, B. Basics of diamond- $\alpha$ partial dynamic calculus on time scales. Math. Comput. Model. 2009, 50, 1253-1261. [CrossRef] 
20. Tian, J.-F.; Zhu, Y.-R.; Cheung, W.-S. N-tuple Diamond-Alpha integral and inequalities on time scales. Rev. R. Acad. Cienc. Exactas Fís. Nat. Ser. A Mat. RACSAM 2019, 113, 2189-2200. [CrossRef]

21. Liu, W.-J.; Tuna, A. Diamond- $\alpha$ weighted Ostrowski type and Grüss type inequalities on time scales. Appl. Math. Comput. 2015, 270, 251-260.

22. Bohner, M.; Matthews, T. Ostrowski inequalities on time scales. J. Inequal. Pure Appl. Math. 2008, 9, 8. [CrossRef]

23. Kermausuor, S.; Nwaeze, E.R. A parameter-based Ostrowski-Grüss type inequalities with multiple points for derivatives bounded by functions on time scales. Mathematics 2018, 6, 326. [CrossRef]

24. Liu, W.-J.; Ngô, Q.A.; Chen, W.-B. Ostrowski type inequalities on time scales for double integrals. Acta. Appl. Math. 2010, 110, 477-497.

25. Du, F.-F.; Hu, W.; Erbe, L. Some new integral inequalities on time scales. Math. Inequal. Appl. 2019, 22, 1-23.

26. Yin, L.; Krasniqi, V. Some generalizations of Feng Qi type integral inequalities on time scales. Appl. Math. ENote 2016, 16, 231-243. [CrossRef]

27. Yin, L.; Luo, Q.-M.; Qi, F. Several integral inequalities on time scales. J. Math. Inequal. 2012, 6, 419-429. [CrossRef]

28. Yin, L.; Qi, F. Some integral inequalities on time scales. Results Math. 2013, 64, 371-381. 